Supporting Information

\title{
Tetrasubstituted Olefin Synthesis via Pd-Catalyzed Addition of Arylboronic Acids to Internal Alkynes Using $\mathrm{O}_{2}$ as an Oxidant
}

\author{
Chengxiang Zhou and Richard C. Larock* \\ Department of Chemistry, Iowa State University, Ames, Iowa 50011 \\ larock@iastate.edu
}

Table of Contents

S1

General Procedures

S2

Characterization data

S3-S7

References

S7

${ }^{1} \mathrm{H}$ and ${ }^{13} \mathrm{C}$ NMR spectra

S8-S33 


\section{General}

The ${ }^{1} \mathrm{H}$ and ${ }^{13} \mathrm{C}$ NMR spectra were recorded at $300 \mathrm{MHz}$ or $400 \mathrm{MHz}$ and $75 \mathrm{MHz}$ or $100 \mathrm{MHz}$, respectively. The COESY and NOESY NMR spectra were recorded at $400 \mathrm{MHz}$. Thin layer chromatography was performed using commercially prepared 60-mesh silica gel plates, and visualization was effected with short wavelength UV light $(254 \mathrm{~nm})$. Low resolution mass spectra were recorded on a triple quadruple mass spectrometer. High resolution mass spectra were recorded on a double focusing magnetic sector mass spectrometer using EI at $70 \mathrm{eV}$. All melting points are uncorrected. All reagents were used as obtained commercially. The product characterization data, ${ }^{1} \mathrm{H}$ and ${ }^{13} \mathrm{C}$ NMR spectra for compounds 4, 5, 7-9, 14, 16-18, 21 can be found in the "Supporting Information" from our previous communication. ${ }^{1}$

\section{General procedure for the synthesis of tetrasubstituted olefins (Table 2, eqs. 5 and 6)}

$\mathrm{Pd}(\mathrm{OAc})_{2}(0.0125 \mathrm{mmol})$, the internal alkyne $(0.25 \mathrm{mmol})$, the arylboronic acid $(1.25$ mmol; $0.5 \mathrm{mmol}$ of $p$-methoxyphenylboronic acid and $0.75 \mathrm{mmol}$ of 3-nitrophenylboronic acid for eq. 6), molecular sieves (Fisher Chemicals, type $4 \AA$, grade 514, 8-12 mesh, beads, $0.2 \mathrm{~g})$, and DMSO $(2 \mathrm{~mL})$ were placed in a 6-dram vial. The vial was sealed with a septum, degassed, and equipped with an $\mathrm{O}_{2}$ balloon. The contents were stirred at room temperature or at $50{ }^{\circ} \mathrm{C}$ for $24 \mathrm{~h}$. The reaction mixture was quenched with $20 \mathrm{~mL}$ of satd $\mathrm{NaCl}$ solution. The resulting mixture was extracted three times with ethyl ether $(30 \mathrm{~mL}$ each time). The combined organic layers were dried over anhydrous $\mathrm{MgSO}_{4}$ and the solvent was evaporated under reduced pressure. The product was isolated by chromatography on a silica gel column.

\section{General procedure for the synthesis of biaryl by homocoupling of aryl bronic acids}

\section{(Table 3).}

$\mathrm{Pd}(\mathrm{OAc})_{2}(0.05 \mathrm{mmol})$, the arylboronic acid $(1.0 \mathrm{mmol})$, molecular sieves (Fisher Chemicals, type $4 \AA$, grade 514, 8-12 mesh, beads, $0.2 \mathrm{~g}$ ), and DMSO (2 mL) were placed in a 6-dram vial. The vial was sealed with a septum, degassed, and equipped with an $\mathrm{O}_{2}$ balloon. The contents were stirred at room temperature or at $50{ }^{\circ} \mathrm{C}$ for $24 \mathrm{~h}$. The reaction mixture was quenched with $20 \mathrm{~mL}$ of satd $\mathrm{NaCl}$ solution. The resulting mixture was extracted three times with ethyl ether $(30 \mathrm{~mL}$ each time $)$. The combined organic layers 
were dried over anhydrous $\mathrm{MgSO}_{4}$ and the solvent was evaporated under reduced pressure. The product was isolated by chromatography on a silica gel column.

\section{Characterization data:}

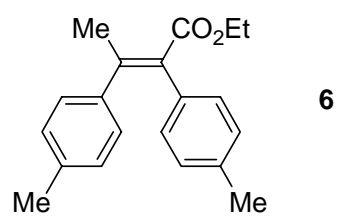

Ethyl (E)-2,3-di-(4-methylphenyl)-butenoate (6) . Colorless liquid: ${ }^{1} \mathrm{H}$ NMR (400 MHz, $\left.\mathrm{CDCl}_{3}\right) \delta 1.31(\mathrm{t}, J=7.2 \mathrm{~Hz}, 3 \mathrm{H}), 2.25(\mathrm{~s}, 3 \mathrm{H}), 2.27(\mathrm{~s}, 3 \mathrm{H}), 2.30(\mathrm{~s}, 3 \mathrm{H}), 4.28(\mathrm{q}, J=7.2$ $\mathrm{Hz}, 2 \mathrm{H}), 6.90-7.00(\mathrm{~m}, 8 \mathrm{H}) ;{ }^{13} \mathrm{C} \mathrm{NMR}\left(100 \mathrm{MHz}, \mathrm{CDCl}_{3}\right) \delta 14.52,21.43,21.44,23.39$, $61.11,128.72,128.81,128.86,129.81,131.83,134.41,136.58,136.90,139.08,142.22$, 170.30; IR $\left(\mathrm{CDCl}_{3}, \mathrm{~cm}^{-1}\right)$ 3022, 2968, 1715, 1598, 1491; MS (EI) m/z (rel intensity) 294 $\left(\mathrm{M}^{+}, 100\right), 248$ (28); HRMS (EI) calcd for $\mathrm{C}_{20} \mathrm{H}_{22} \mathrm{O}_{2}$ 294.1620, found: 294.1624.

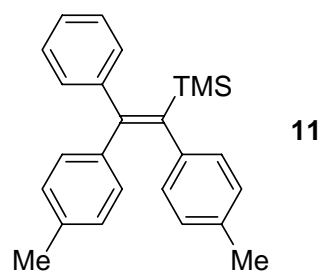

(E)-1,2-Di-(4-methylphenyl)-1-trimethylsilyl-2-phenylethene (11). White solid: mp 88$90{ }^{\circ} \mathrm{C} ;{ }^{1} \mathrm{H}$ NMR $\left(300 \mathrm{MHz}, \mathrm{CDCl}_{3}\right) \delta-0.21(\mathrm{~s}, 9 \mathrm{H}), 2.16(\mathrm{~s}, 3 \mathrm{H}), 2.26(\mathrm{~s}, 3 \mathrm{H}), 6.82-6.96$ $(\mathrm{m}, 8 \mathrm{H}), 7.25-7.32(\mathrm{~m}, 5 \mathrm{H}) ;{ }^{13} \mathrm{C} \mathrm{NMR}\left(75 \mathrm{MHz}, \mathrm{CDCl}_{3}\right) \delta 0.01,20.62,20.66,126.47$, 127.43 , 127.52, 127.69, 128.67, 128.91, 129.08, 133.64, 134.89, 139.91, 140.49, 143.17, 144.26, 152.80; IR $\left(\mathrm{CDCl}_{3}, \mathrm{~cm}^{-1}\right)$ 3026, 2955, 1492; MS (EI) m/z (rel intensity) $356\left(\mathrm{M}^{+}\right.$, 100), 341 (86), 284 (8); HRMS (EI) calcd for $\mathrm{C}_{25} \mathrm{H}_{28}$ Si 356.1960, found: 356.1965.

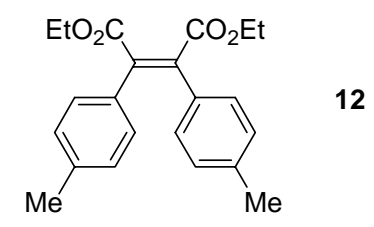

Diethyl (Z)-2,3-di-(4-methylphenyl)-maleate (12). Yellow oil: ${ }^{1} \mathrm{H}$ NMR (400 MHz, $\left.\mathrm{CDCl}_{3}\right) \delta 1.29(\mathrm{t}, J=7.2 \mathrm{~Hz}, 6 \mathrm{H}), 2.27(\mathrm{~s}, 6 \mathrm{H}), 4.28(\mathrm{q}, J=7.2 \mathrm{~Hz}, 4 \mathrm{H}), 6.97-7.99(\mathrm{~m}, 8 \mathrm{H})$; ${ }^{13} \mathrm{C}$ NMR $\left(75 \mathrm{MHz}, \mathrm{CDCl}_{3}\right) \delta 14.31,21.58,61.86,129.09,129.89,132.02,138.27,138.32$, 168.57; IR $\left(\mathrm{CDCl}_{3}, \mathrm{~cm}^{-1}\right)$ 3058, 2983, 1716, 1492; MS (EI) m/z (rel intensity) $352\left(\mathrm{M}^{+}\right.$, 100), 337 (11), 307 (48); HRMS (EI) calcd for $\mathrm{C}_{22} \mathrm{H}_{24} \mathrm{O}_{4} 352.1675$, found: 352.1680 . 


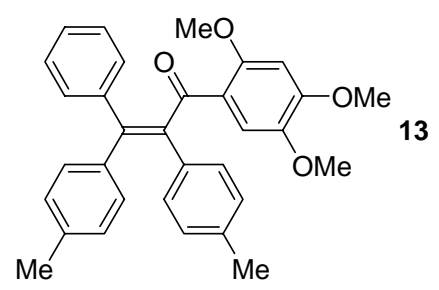

(E)- 1-(2,4,5-Trimethoxyphenyl)-2,3-di-(4-methylphenyl)-3-phenyl-2-propen-1-one (13). Yellow solid: mp 105-107 ${ }^{\circ} \mathrm{C}$; ${ }^{1} \mathrm{H}$ NMR (400 MHz, $\left.\mathrm{CDCl}_{3}\right) \delta 2.23$ (s, 3H), 2.28 (s, 3H), $3.79(\mathrm{~s}, 6 \mathrm{H}), 3.85(\mathrm{~s}, 3 \mathrm{H}), 6.28(\mathrm{~s}, 1 \mathrm{H}), 6.89-7.01(\mathrm{~m}, 8 \mathrm{H}), 7.09-7.15(\mathrm{~m}, 5 \mathrm{H}), 7.22(\mathrm{~s}$, $1 \mathrm{H}) ;{ }^{13} \mathrm{C} \mathrm{NMR}\left(100 \mathrm{MHz}, \mathrm{CDCl}_{3}\right) \delta 21.49,21.51,56.17,56.20,56.43,95.90,113.77$, $120.37,127.62$, 128.02, 128.69, 128.72, 130.19, 130.58, 131.27, 135.89, 136.60, 137.05, 139.20, 141.44, 142.89, 142.97, 143.15, 153.88, 154.49, 197.23; IR $\left(\mathrm{CDCl}_{3}, \mathrm{~cm}^{-1}\right)$ 3025, 1664, 1598, 1492; MS (EI) m/z (rel intensity) $478\left(\mathrm{M}^{+}, 20\right), 195$ (100); HRMS (EI) calcd for $\mathrm{C}_{32} \mathrm{H}_{30} \mathrm{O}_{4} 478.2144$, found: 478.2152 .

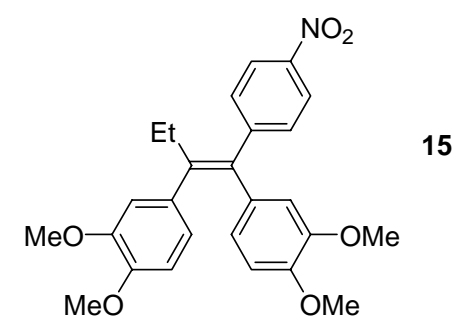

(E)-1,2-Di-(3,4-dimethoxyphenyl)-1-(4-nitrophenyl)-1-butene (15). Yellow oil; ${ }^{1} \mathrm{H}$ $\operatorname{NMR}\left(400 \mathrm{MHz}, \mathrm{CDCl}_{3}\right) \delta 0.98(\mathrm{t}, J=7.2 \mathrm{~Hz}, 3 \mathrm{H}), 2.43(\mathrm{q}, J=7.6 \mathrm{~Hz}, 2 \mathrm{H}), 3.49(\mathrm{~s}, 3 \mathrm{H})$, $3.63(\mathrm{~s}, 3 \mathrm{H}), 3.76(\mathrm{~s}, 3 \mathrm{H}), 3.83(\mathrm{~s}, 3 \mathrm{H}), 6.35-6.38(\mathrm{~m}, 2 \mathrm{H}), 6.56-6.60(\mathrm{~m}, 2 \mathrm{H}), 6.72-6.73(\mathrm{~m}$, 2H), $7.40(\mathrm{~d}, J=8.8 \mathrm{~Hz}, 2 \mathrm{H}), 8.20(\mathrm{~d}, J=8.8 \mathrm{~Hz}, 2 \mathrm{H}) ;{ }^{13} \mathrm{C} \mathrm{NMR}\left(75 \mathrm{MHz}, \mathrm{CDCl}_{3}\right) \delta$ 13.90, 29.03, 55.81, 55.93, 56.00, 56.03, 110.60, 110.98, 113.46, 114.47, 121.65, 123.49, $123.73,130.61,134.15,134.97,136.41,143.57,146.71,147.70,148.03,148.24,148.71$, 150.97; IR $\left(\mathrm{CDCl}_{3}, \mathrm{~cm}^{-1}\right)$ 3022, 2960, 1598; MS (EI) m/z (rel intensity) $449\left(\mathrm{M}^{+}, 100\right)$, 434 (12); HRMS (EI) calcd for $\mathrm{C}_{26} \mathrm{H}_{27} \mathrm{NO}_{6} 449.1838$, found: 449.1842.

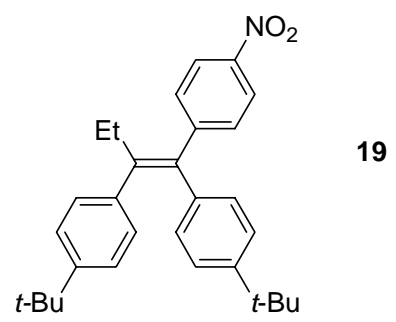

(E)-1,2-Di-(4-t-butylphenyl)-1-(4-nitrophenyl)-1-butene (19). Light yellow solid (mp 135-137 $\left.{ }^{\circ} \mathrm{C}\right):{ }^{1} \mathrm{H}$ NMR (400 MHz, $\left.\mathrm{CDCl}_{3}\right) \delta 0.96(\mathrm{t}, J=7.6 \mathrm{~Hz}, 3 \mathrm{H}), 1.20(\mathrm{~s}, 9 \mathrm{H}), 1.35$ (s, 
9H), 2.43 (q, $J=7.6 \mathrm{~Hz}, 2 \mathrm{H}), 6.70-6.73(\mathrm{~m}, 2 \mathrm{H}), 7.00-7.03(\mathrm{~m}, 4 \mathrm{H}), 7.15-7.18(\mathrm{~m}, 2 \mathrm{H})$, $7.42(\mathrm{dd}, J=1.6,8.8 \mathrm{~Hz}, 2 \mathrm{H}), 8.22(\mathrm{dd}, J=1.6,8.8 \mathrm{~Hz}, 2 \mathrm{H}) ;{ }^{13} \mathrm{C} \mathrm{NMR}\left(100 \mathrm{MHz}, \mathrm{CDCl}_{3}\right)$ $\delta 13.92,29.19,31.45,31.54,34.57,34.66,123.72,124.61,124.89,129.29,130.53,130.69$, 136.77, 138.43, 139.03, 144.14, 146.61, 149.29, 149.71, 151.17; IR ( $\left.\mathrm{CDCl}_{3}, \mathrm{~cm}^{-1}\right) 3058$, 2966, 1492; MS (EI) m/z (rel intensity) $441\left(\mathrm{M}^{+}, 100\right), 416$ (16), 279 (80); HRMS (EI) calcd for $\mathrm{C}_{30} \mathrm{H}_{35} \mathrm{NO}_{2} 441.2668$, found: 441.2674 .

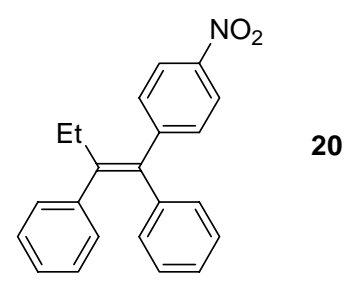

(E)-1-(4-Nitrophenyl)-1,2-diphenyl-1-butene (20). Light yellow solid: mp 140-142 ${ }^{\circ} \mathrm{C}$; ${ }^{1} \mathrm{H}$ NMR $\left(300 \mathrm{MHz}, \mathrm{CDCl}_{3}\right) \delta 0.99(\mathrm{t}, J=7.2 \mathrm{~Hz}, 3 \mathrm{H}), 2.48(\mathrm{q}, J=7.5 \mathrm{~Hz}, 2 \mathrm{H}), 6.85-6.88$ (m, 2H), 7.04-7.20 (m, 8H), 7.42-7.45 (m, 2H), 8.22-8.25 (m, 2H) ; ${ }^{13} \mathrm{C} \mathrm{NMR} \mathrm{(100} \mathrm{MHz,}$ $\left.\mathrm{CDCl}_{3}\right) \delta 13.78,29.30,123.82,126.66,126.98,127.98,128.24,129.72,130.66,130.98$, 137.17, 141.39, 141.98, 144.62, 146.77, 150.73; IR $\left(\mathrm{CDCl}_{3}, \mathrm{~cm}^{-1}\right) 3022,1516,1442$; MS (EI) $\mathrm{m} / \mathrm{z}$ (rel intensity) $329\left(\mathrm{M}^{+}, 100\right), 300$ (21), 253 (28); HRMS (EI) calcd for $\mathrm{C}_{22} \mathrm{H}_{19} \mathrm{NO}_{2}$ 329.1416, found: 329.1422 .

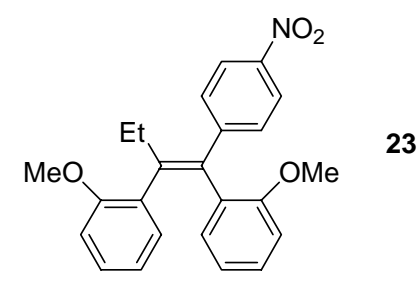

(E)-1,2-Di-(2-methoxyphenyl)-1-(4-nitrophenyl)-1-butene (23). Light yellow oil: ${ }^{1} \mathrm{H}$ NMR (400 MHz, $\left.\mathrm{CDCl}_{3}\right) \delta 0.99(\mathrm{t}, J=7.2 \mathrm{~Hz}, 3 \mathrm{H}), 2.50(\mathrm{br}, 2 \mathrm{H})$ (the absence of the quartet of the $-\mathrm{CH}_{2}$ - group is possibly due to the steric bulkiness around this group), 3.56 (s, $3 \mathrm{H}), 3.77$ (s, 3H), 6.61-6.76 (m, 4H), 6.90-7.10 (m, 4H), 7.47-7.50 (m, 2H), 8.13-8.18 (m, $2 \mathrm{H}) ;{ }^{13} \mathrm{C} \mathrm{NMR}\left(100 \mathrm{MHz}, \mathrm{CDCl}_{3}\right) \delta 13.79,26.85,55.36,55.39,110.31,110.81,120.03$, $120.17,122.54,123.21,128.17,128.52,130.35,130.68,130.78,131.07,131.74,134.97$, 146.33, 150.07, 156.84, 156.97; IR $\left(\mathrm{CDCl}_{3}, \mathrm{~cm}^{-1}\right)$ 3023, 2960, 2870, 1493; MS (EI) m/z (rel intensity) $389\left(\mathrm{M}^{+}, 100\right), 374$ (8); HRMS (EI) calcd for $\mathrm{C}_{24} \mathrm{H}_{23} \mathrm{NO}_{4}$ 389.1627, found: 389.1632. 


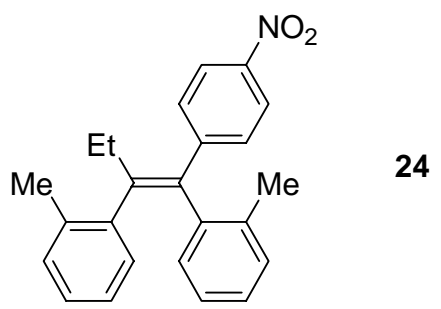

(E)-1,2-Di-(2-methylphenyl)-1-(4-nitrophenyl)-1-butene (24). Light yellow oil: ${ }^{1} \mathrm{H}$ NMR (300 MHz, CDCl $\left.)_{3}\right) \delta 1.05$ (t, J=7.2 Hz, 3H), 2.03 (br, 3H), 2.25 (br, 3H), 2.53 (br, $2 \mathrm{H})$ (the appearance of the broad peaks and the absence of the quartet of $-\mathrm{CH}_{2}$ - group are possibly due to the steric bulkiness around these groups), 6.88-7.02 (m, 8H), 7.42-7.47 (m, $2 \mathrm{H}), 8.16-8.21(\mathrm{~m}, 2 \mathrm{H}) ;{ }^{13} \mathrm{C} \mathrm{NMR}\left(75 \mathrm{MHz}, \mathrm{CDCl}_{3}\right) \delta 13.63,20.13,20.76,123.54,125.17$, $125.35,126.92,127.37,129.56,130.10,130.43,135.99,140.72,146.43$ (the absence of some carbon signals is possibly due to the steric bulkiness around these carbons and possible overlap of some carbon signals); IR $\left(\mathrm{CDCl}_{3}, \mathrm{~cm}^{-1}\right) 3027,2928,1508,1490$; MS (EI) $\mathrm{m} / \mathrm{z}$ (rel intensity) $357\left(\mathrm{M}^{+}, 100\right), 342$ (11), 267 (20); HRMS (EI) calcd for $\mathrm{C}_{24} \mathrm{H}_{23} \mathrm{NO}_{2}$ 357.1729, found: 357.1736 .

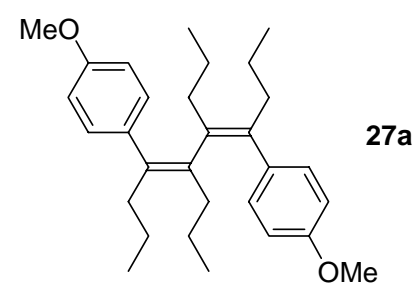

(Z,Z)-4,7-Di-(4-methoxyphenyl)-5,6-dipropyl-4,6-decadiene (27a). Light yellow oil: ${ }^{1} \mathrm{H}$ NMR (400 M Hz, $\left.\mathrm{CDCl}_{3}\right) \delta$ 0.77-0.83 (m, 12H), 1.16-1.40 (m, 8H), 1.55-1.58 (m, 2H), 1.99-2.03 (m, 2H), 2.18-2.30 (m, 4H), 3.81 (s, 6H), 6.75 (d, $J=8.8 \mathrm{~Hz}, 4 \mathrm{H}), 7.10$ (d, $J=$ $8.8 \mathrm{~Hz}, 4 \mathrm{H}) ;{ }^{13} \mathrm{C} \mathrm{NMR}\left(100 \mathrm{M} \mathrm{Hz}, \mathrm{CDCl}_{3}\right) \delta 14.44,15.11,21.73,22.98,36.21,36.57$, 55.40, 112.95, 129.78, 136.05, 136.52, 139.19, 157.72; IR $\left(\mathrm{CDCl}_{3}, \mathrm{~cm}^{-1}\right) 3023,2958,2930$, 2871, 1601, 1492; MS (EI) m/z (rel intensity) 434 (M+1, 65), 121 (100); HRMS (EI) calcd for $\mathrm{C}_{30} \mathrm{H}_{42} \mathrm{O}_{2}$ 434.3185, found: 434.3190 .

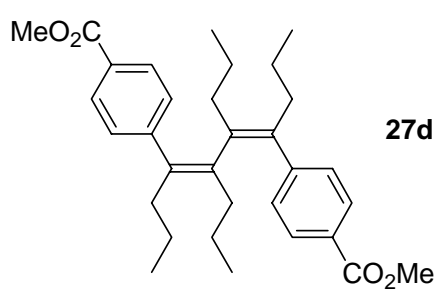

Dimethyl (Z,Z)-4,4'-(1,3-butadiene-1,4-diyl)-1,2,3,4-tetrapropyl-bis-benzoate(27d). Colorless liquid: ${ }^{1} \mathrm{H}$ NMR (400 MHz, $\left.\mathrm{CDCl}_{3}\right) \delta$ 0.72-0.77 (m, 6H), 0.88-0.95 (m, 6H), 
1.05-1.11 (m, 4H), 1.40-1.50 (m, 4H), 1.75-1.85 (m, 2H), 2.10-2.25 (m, 6H), $3.91(\mathrm{~s}, 6 \mathrm{H})$, 6.91-6.96 (m, 4H), 7.80-7.86 (m, 4H); $\left.{ }^{13} \mathrm{C} \mathrm{NMR} \mathrm{(100} \mathrm{MHz,} \mathrm{CDCl}_{3}\right) \delta 14.29,15.11,21.68$, $23.12,35.83,36.82,52.20,127.55,128.85,128.92,137.12,140.15,148.92,167.51$; IR $\left(\mathrm{CDCl}_{3}, \mathrm{~cm}^{-1}\right)$ 3026, 1710, 1599, 1491; MS (EI) m/z (rel intensity) $490\left(\mathrm{M}^{+}, 56\right), 448(98)$, 415 (82), 163 (100); HRMS (EI) calcd for $\mathrm{C}_{31} \mathrm{H}_{42} \mathrm{O}_{4} 490.3083$, found: 490.3090.

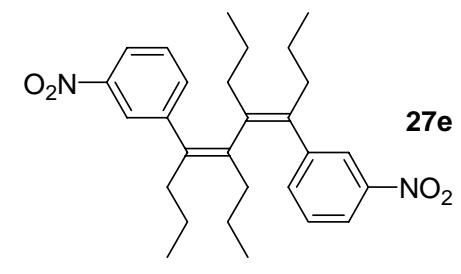

(Z,Z)-4,7-Di-(3-nitrophenyl)-5,6-dipropyl-4,6-decadiene (27e). Light yellow liquid: ${ }^{1} \mathrm{H}$ NMR (400 MHz, $\left.\mathrm{CDCl}_{3}\right) \delta$ 0.72-0.79 (m, 6H), 0.90-1.10 (m, 10H), 1.56-1.66(m, 4H), 1.92-2.33 (m, 8H), 6.89-6.93 (m, 2H), 7.20-7.28 (m, 2H), 7.46-7.48 (m, 2H), 7.95-8.00 (m, $2 \mathrm{H}) ;{ }^{13} \mathrm{C}$ NMR $\left(100 \mathrm{MHz}, \mathrm{CDCl}_{3}\right) \delta$ 14.24, 15.30, 21.90, 23.27, 35.62, 36.35, 121.09, 123.71, 128.29, 134.82, 136.52, 140.29, 144.95, 147.69; IR $\left(\mathrm{CDCl}_{3}, \mathrm{~cm}^{-1}\right)$ 3026, 2960, 2928, 2871, 1600, 1491; MS (EI) m/z (rel intensity) $464\left(\mathrm{M}^{+}, 100\right), 421$ (42), 286 (50); HRMS (EI) calcd for $\mathrm{C}_{28} \mathrm{H}_{36} \mathrm{~N}_{2} \mathrm{O}_{4} 464.2675$, found: 464.2683 .

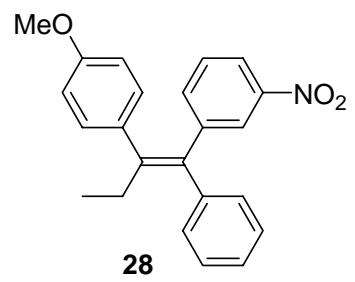

(E)-2-(4-Methoxyphenyl)-1-(3-nitrophenyl)-1-phenyl-1-butene (28). Light yellow liquid: ${ }^{1} \mathrm{H} \mathrm{NMR}\left(400 \mathrm{MHz}, \mathrm{CDCl}_{3}\right) \delta 0.95(\mathrm{t}, J=7.2 \mathrm{~Hz}, 3 \mathrm{H}), 2.48(\mathrm{q}, J=7.2 \mathrm{~Hz}, 2 \mathrm{H})$, $3.75(\mathrm{~s}, 3 \mathrm{H}), 6.71-6.75(\mathrm{~m}, 2 \mathrm{H}), 7.00-7.05(\mathrm{~m}, 2 \mathrm{H}), 7.15-7.38(\mathrm{~m}, 7 \mathrm{H}), 7.76(\mathrm{~s}, 1 \mathrm{H}), 7.83-$ $7.88(\mathrm{~m}, 1 \mathrm{H}) ;{ }^{13} \mathrm{C}$ NMR $\left(100 \mathrm{MHz}, \mathrm{CDCl}_{3}\right) \delta 13.74,29.33,55.39,113.86,120.93,125.74$, $127.40,128.50,128.75,129.71,130.93,133.33$, 136.39, 137.10, 142.44, 144.75, 145.34, 147.93, 158.56; IR ( $\left.\mathrm{CDCl}_{3}, \mathrm{~cm}^{-1}\right)$ 3026, 2930, 1599, 1492; MS (EI) m/z (rel intensity) 359 $\left(\mathrm{M}^{+}, 100\right), 344$ (11); HRMS (EI) calcd for $\mathrm{C}_{23} \mathrm{H}_{21} \mathrm{NO}_{3}$ 359.1521, found: 359.1526 .

\section{References}

(1) Zhou, C.; Larock, R. C. Org. Lett. 2005, 7, 259. 


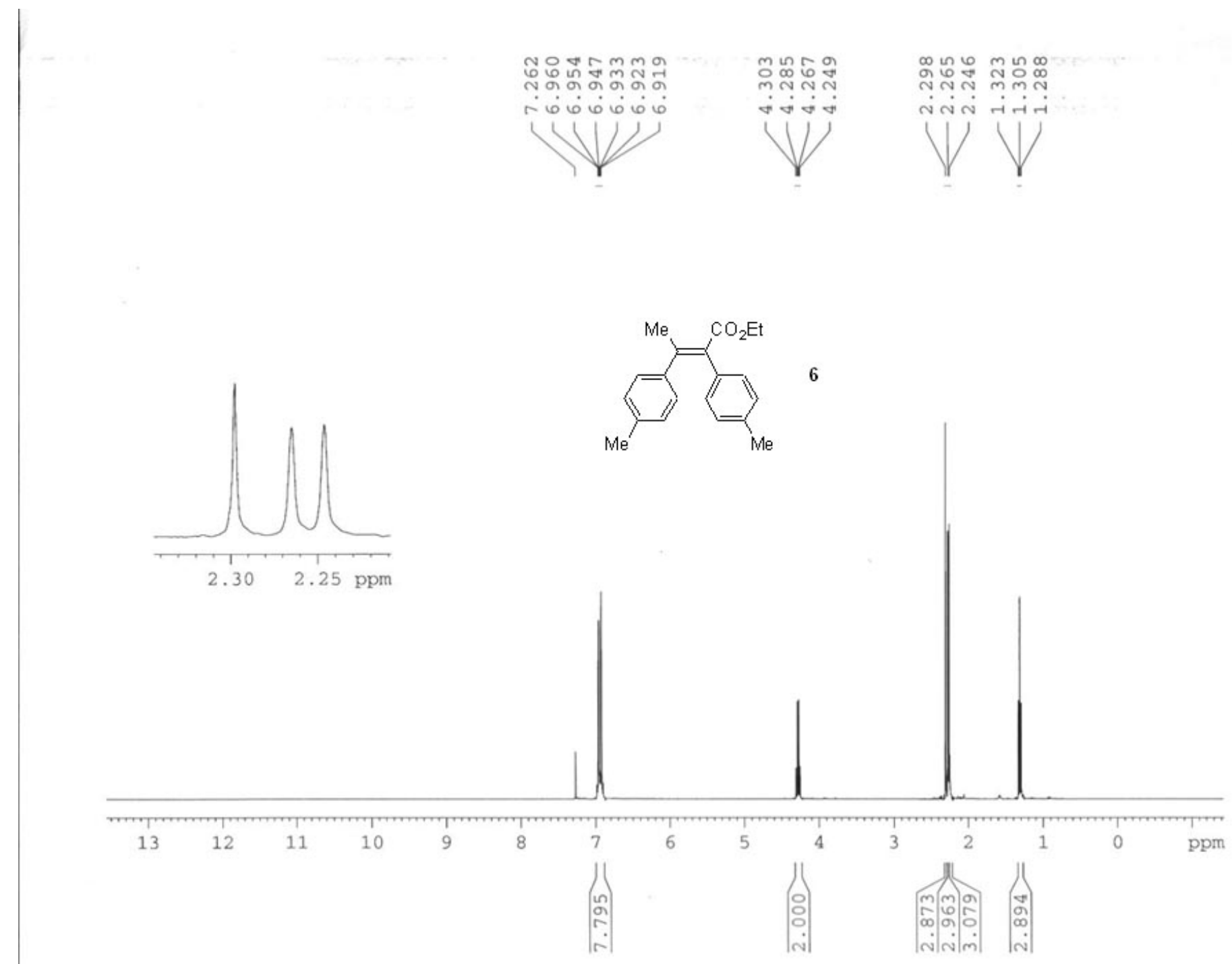

S8 

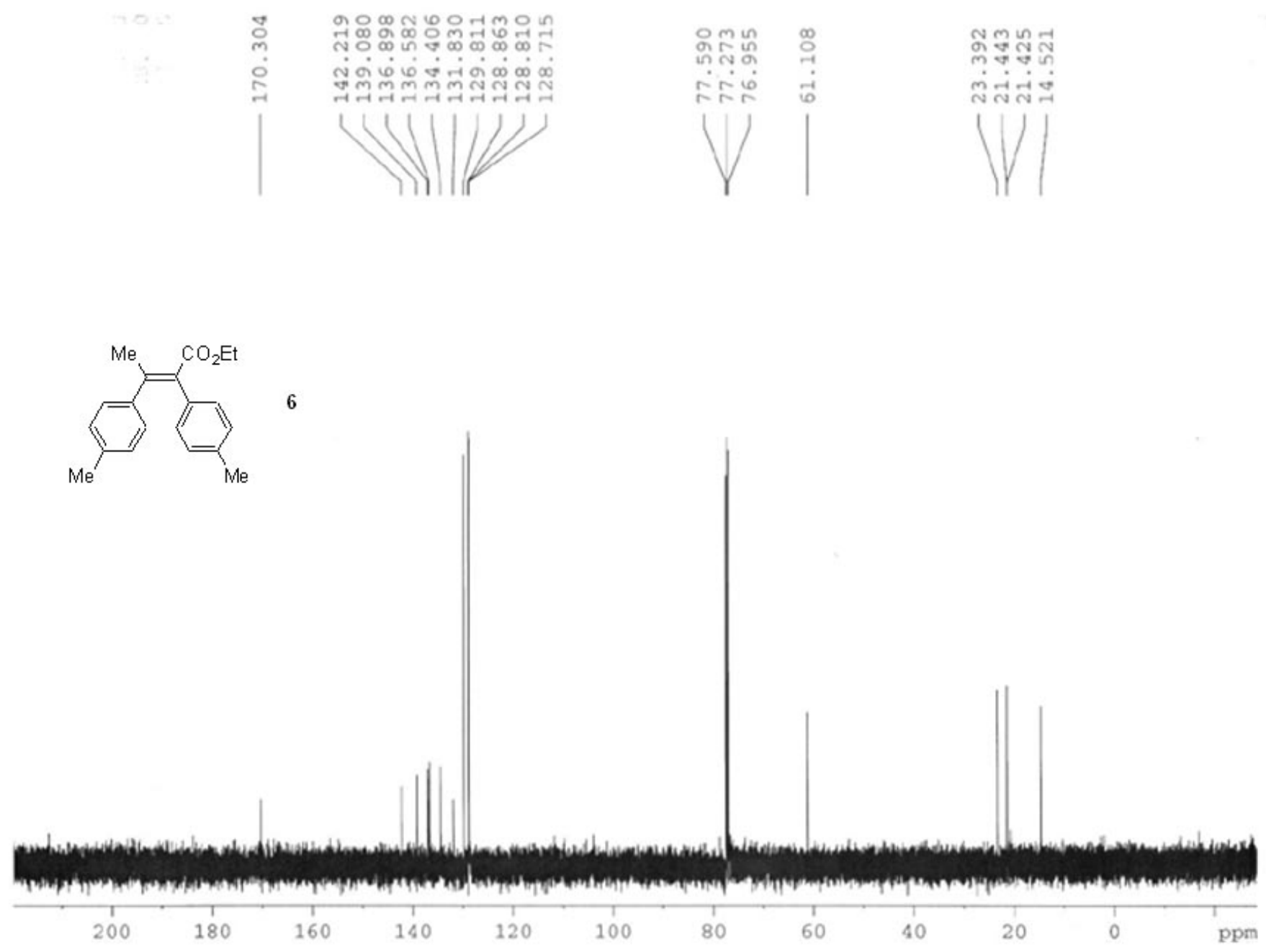
ㅁำ

IVW

$\prod^{11}$
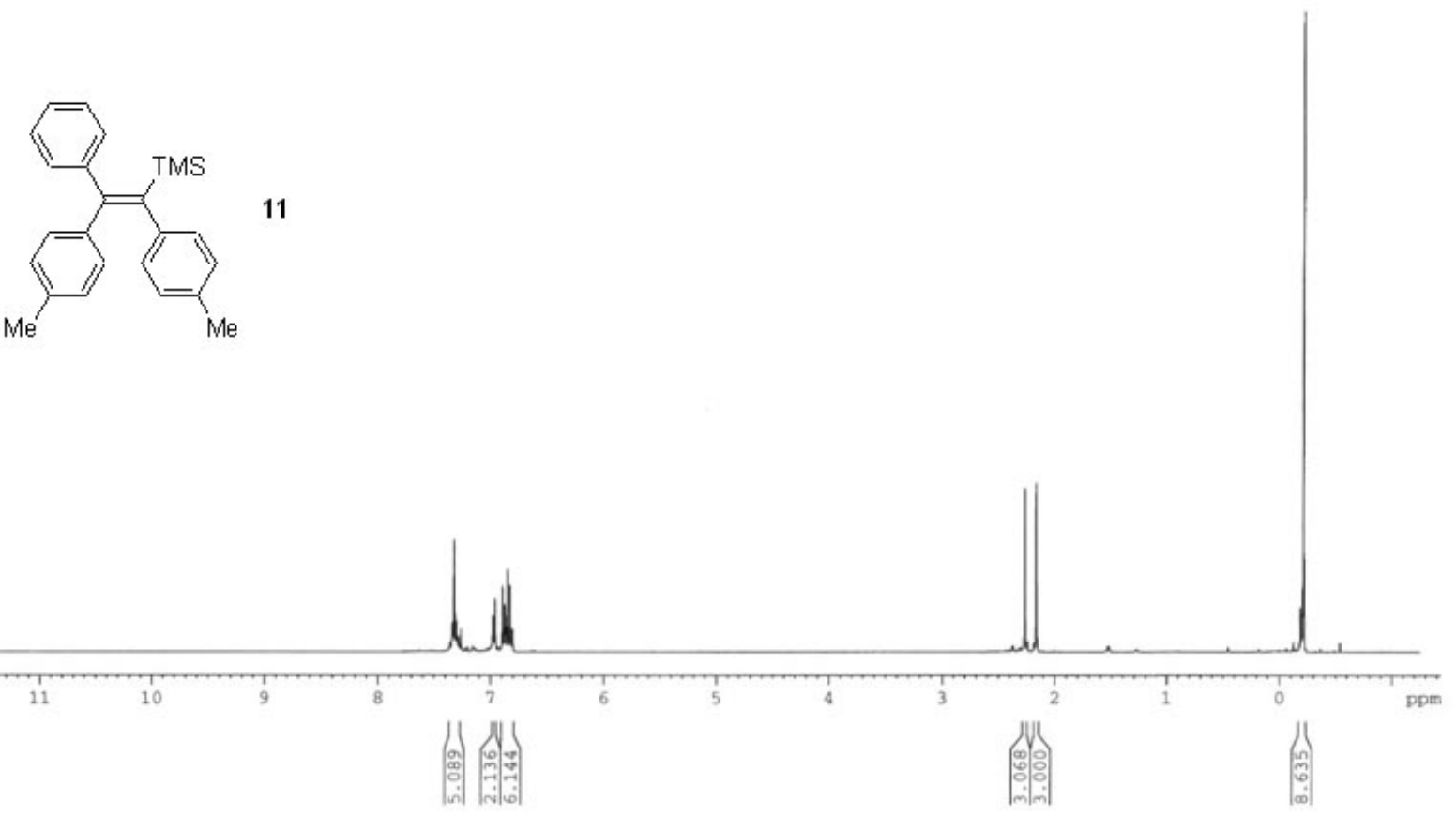


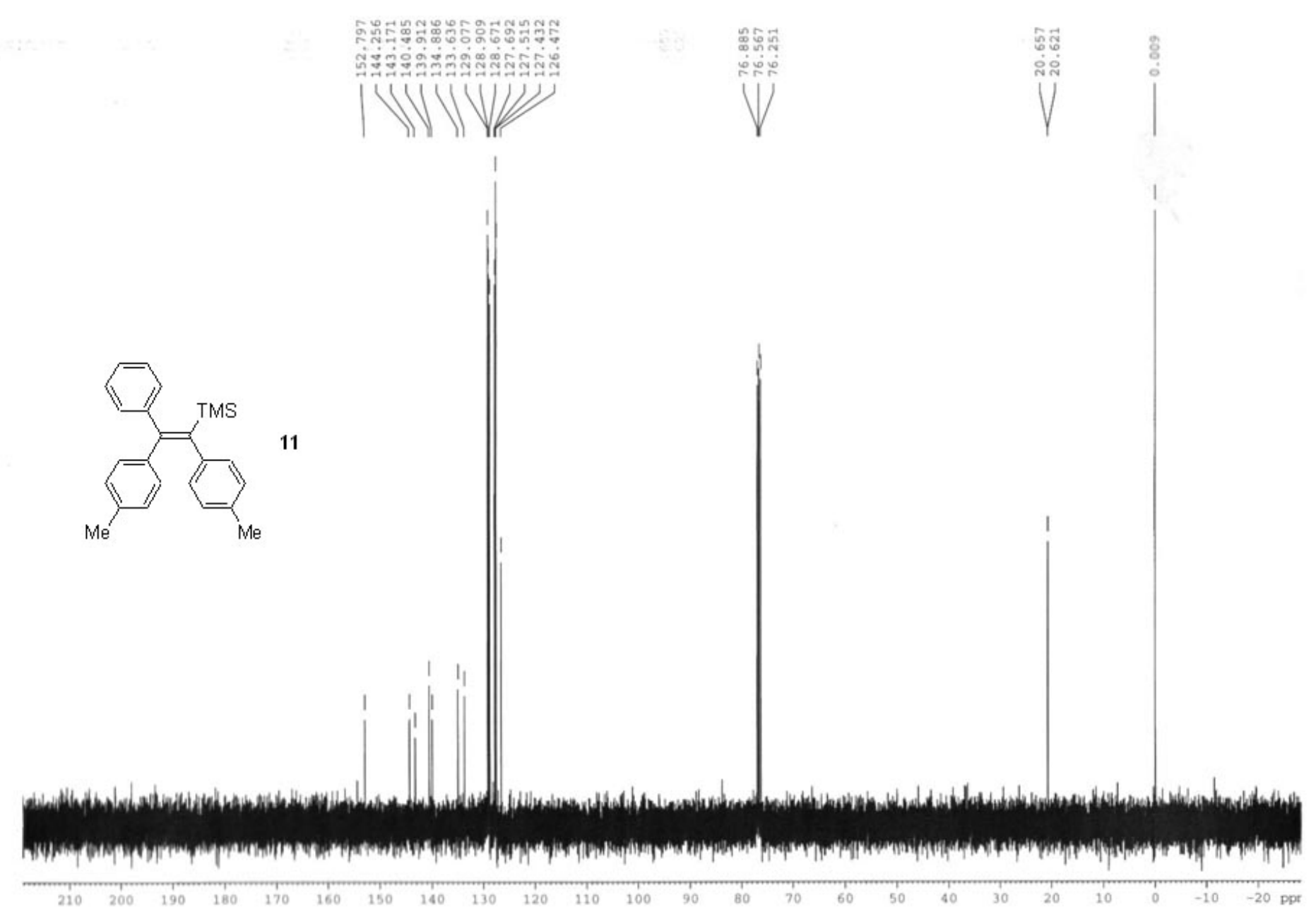



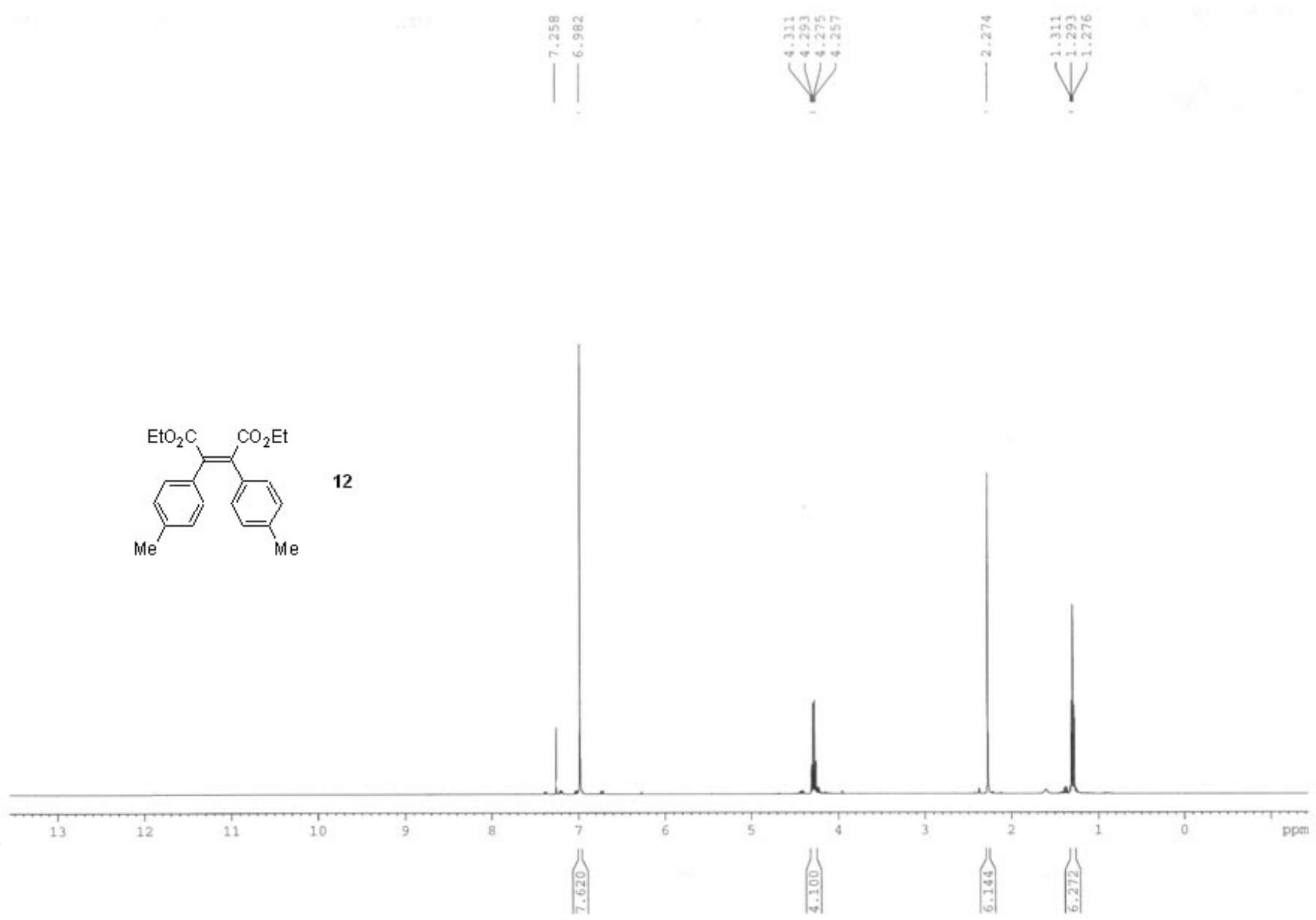

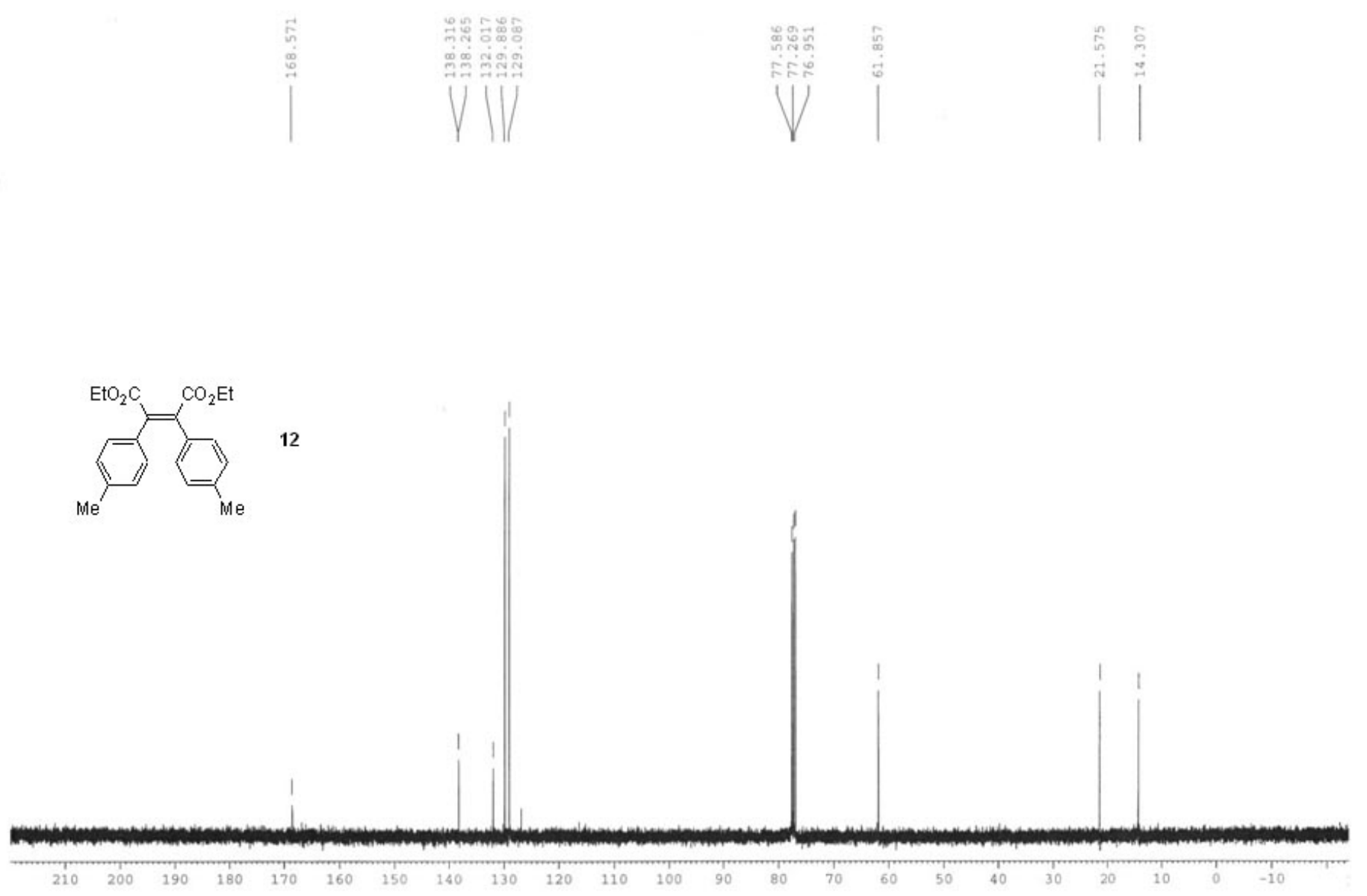

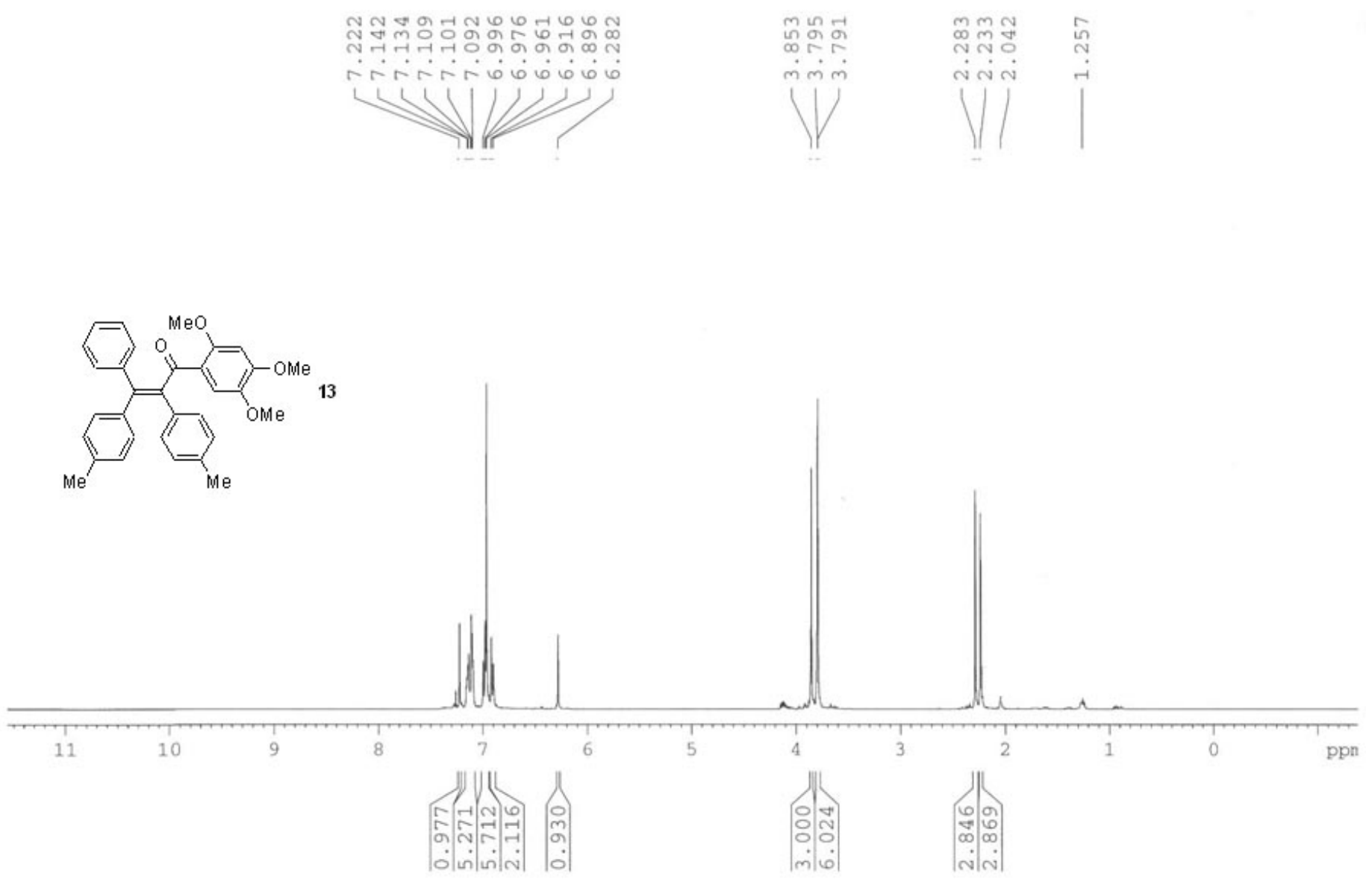


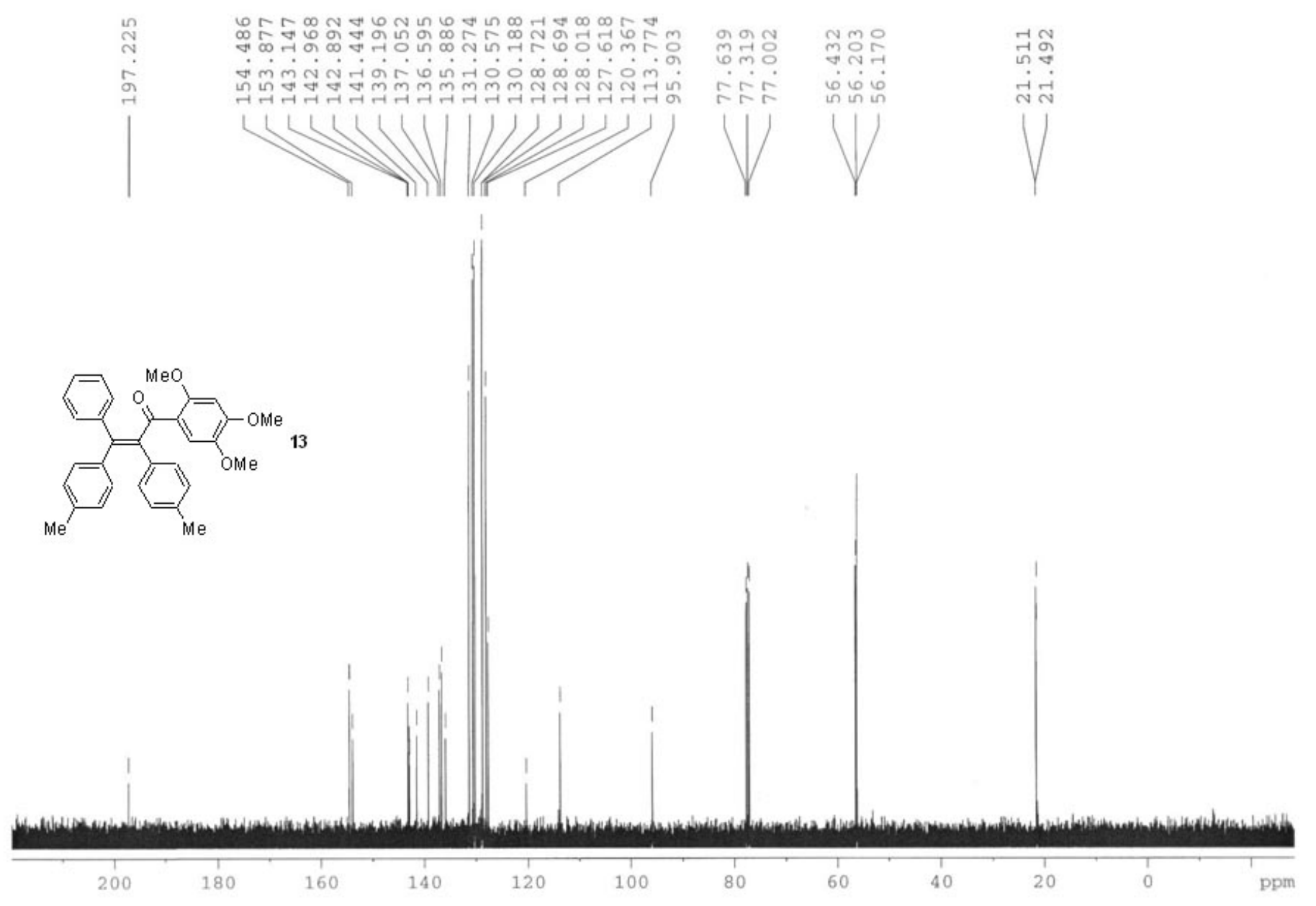



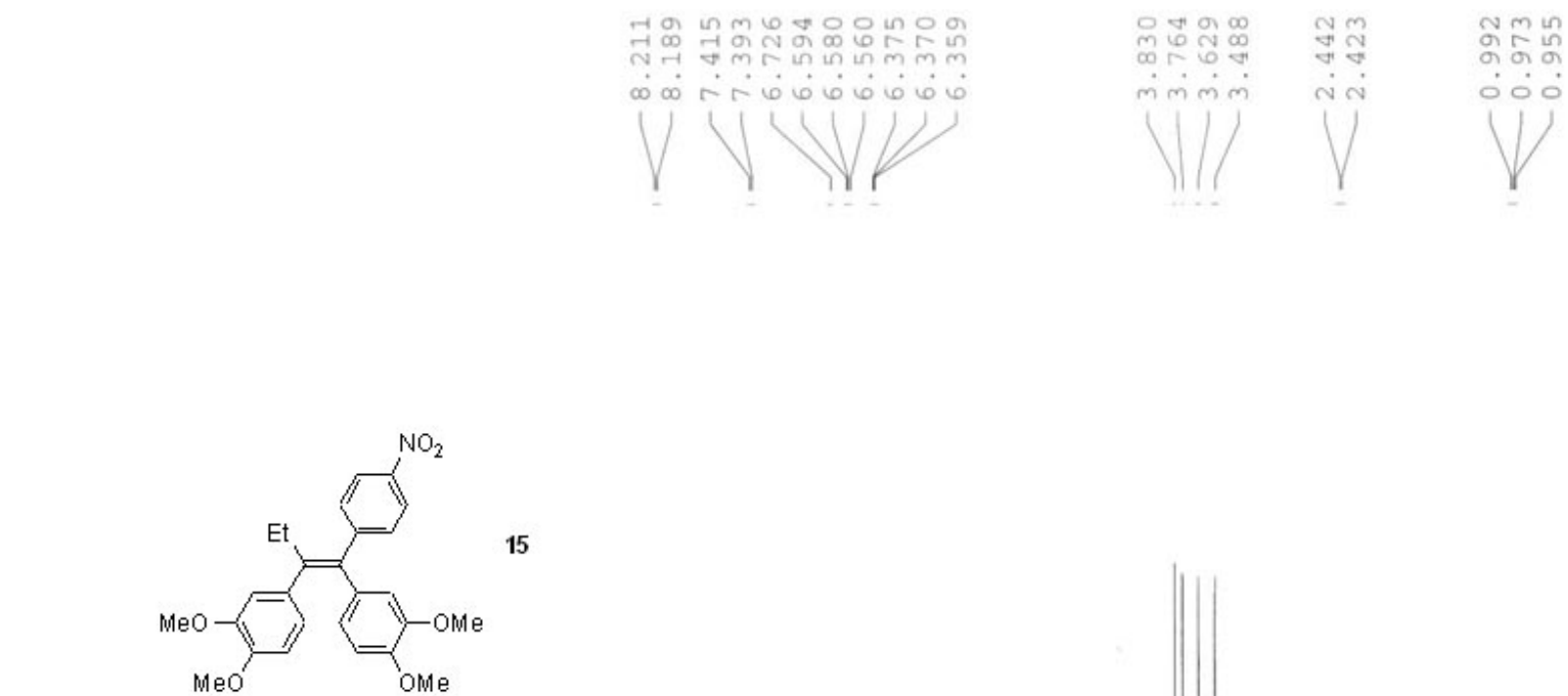

15

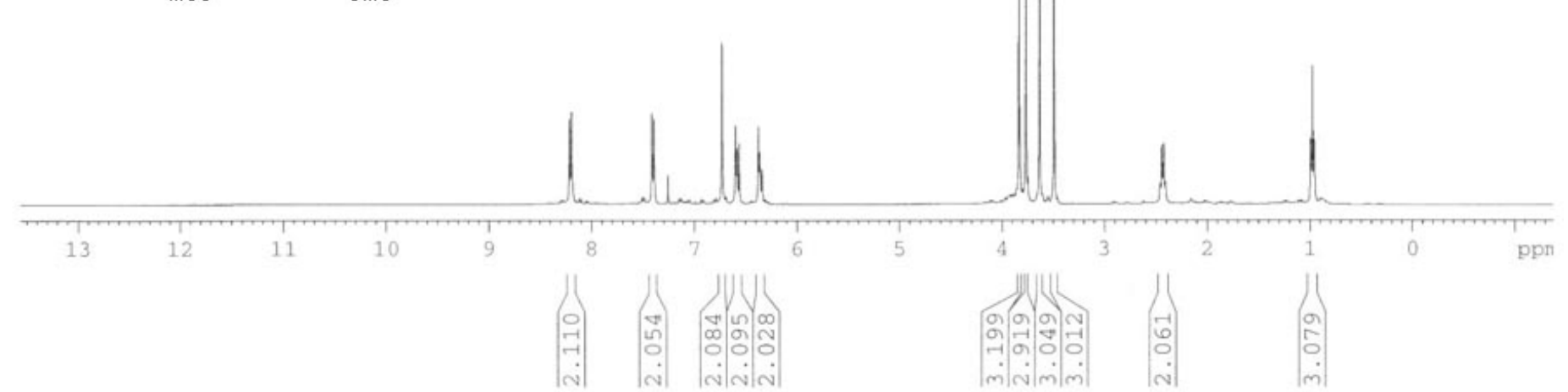



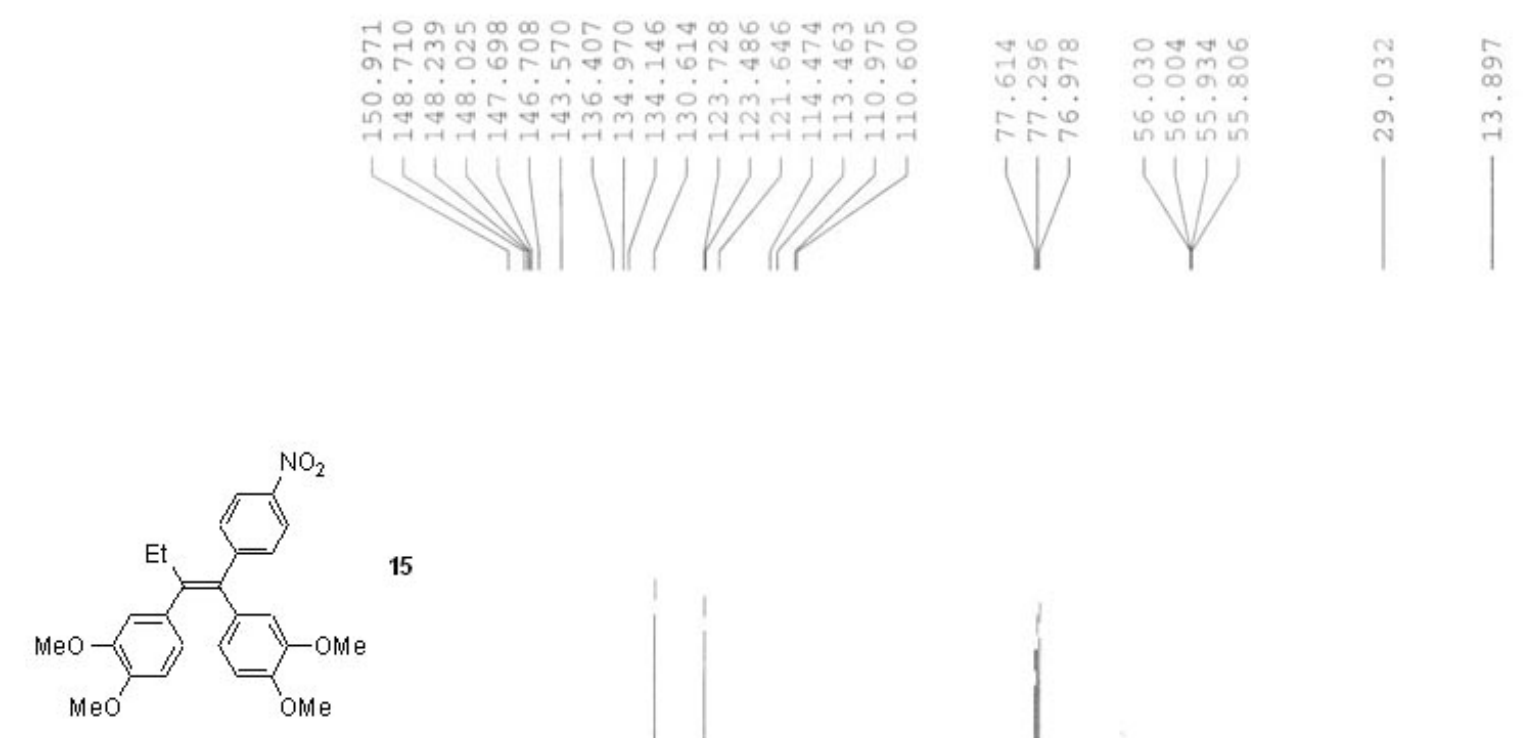

15

$\underset{\text { Meo }}{=} \underset{\text { Ome }}{\longrightarrow \text { OMe }}$

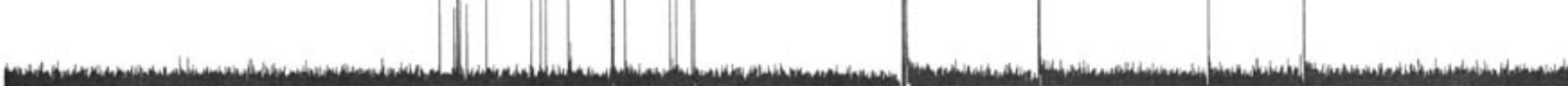

200

180

160

140

120

100

80

60

40

20 


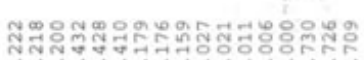

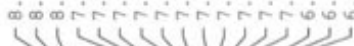
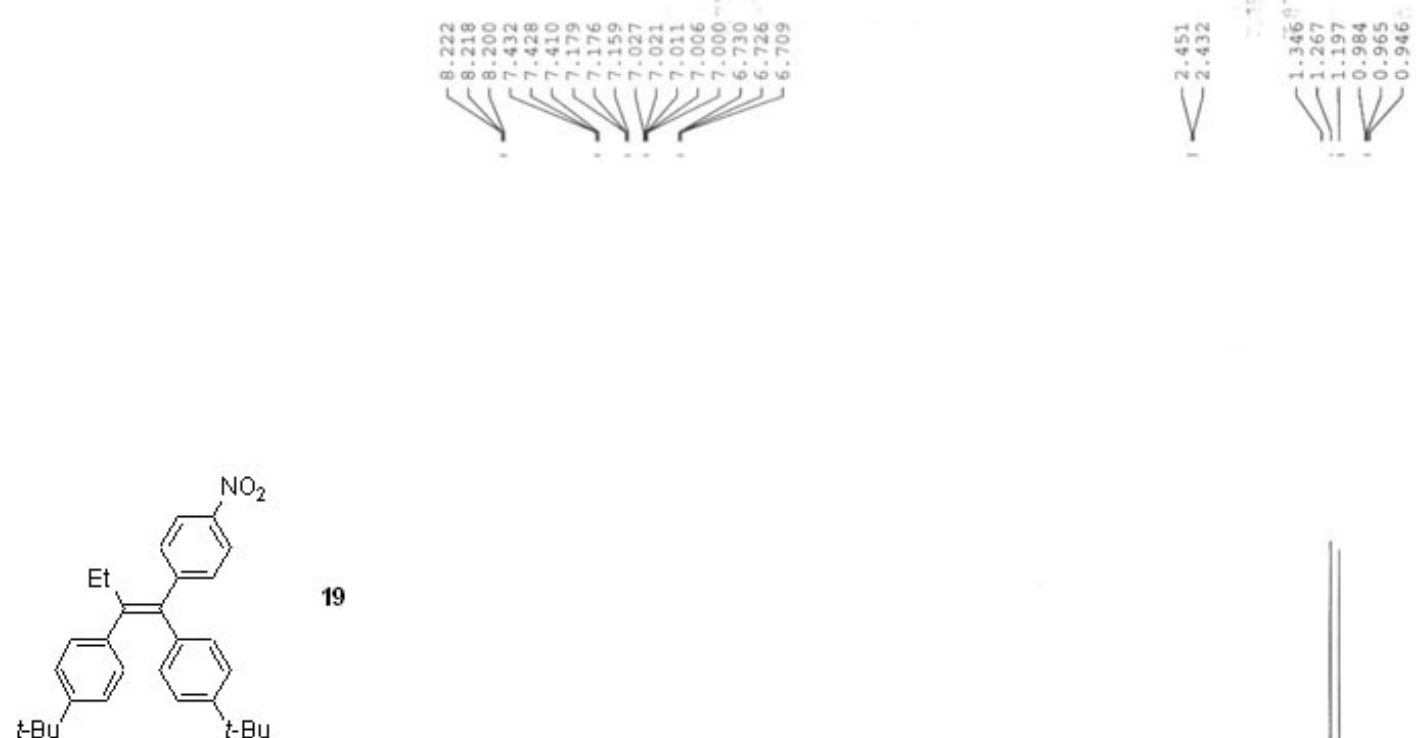

19

${ }_{\mathrm{t}-\mathrm{Bu}}>_{\mathrm{t}-\mathrm{Bu}}$

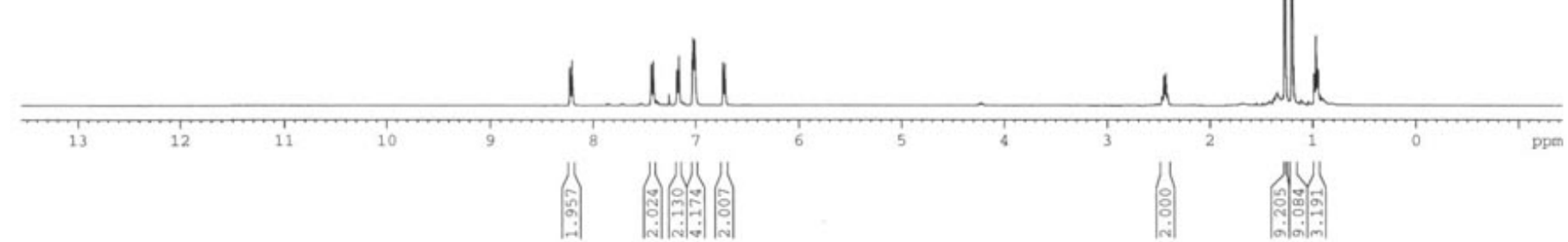




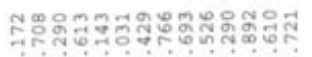

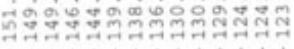

VIIV VWV
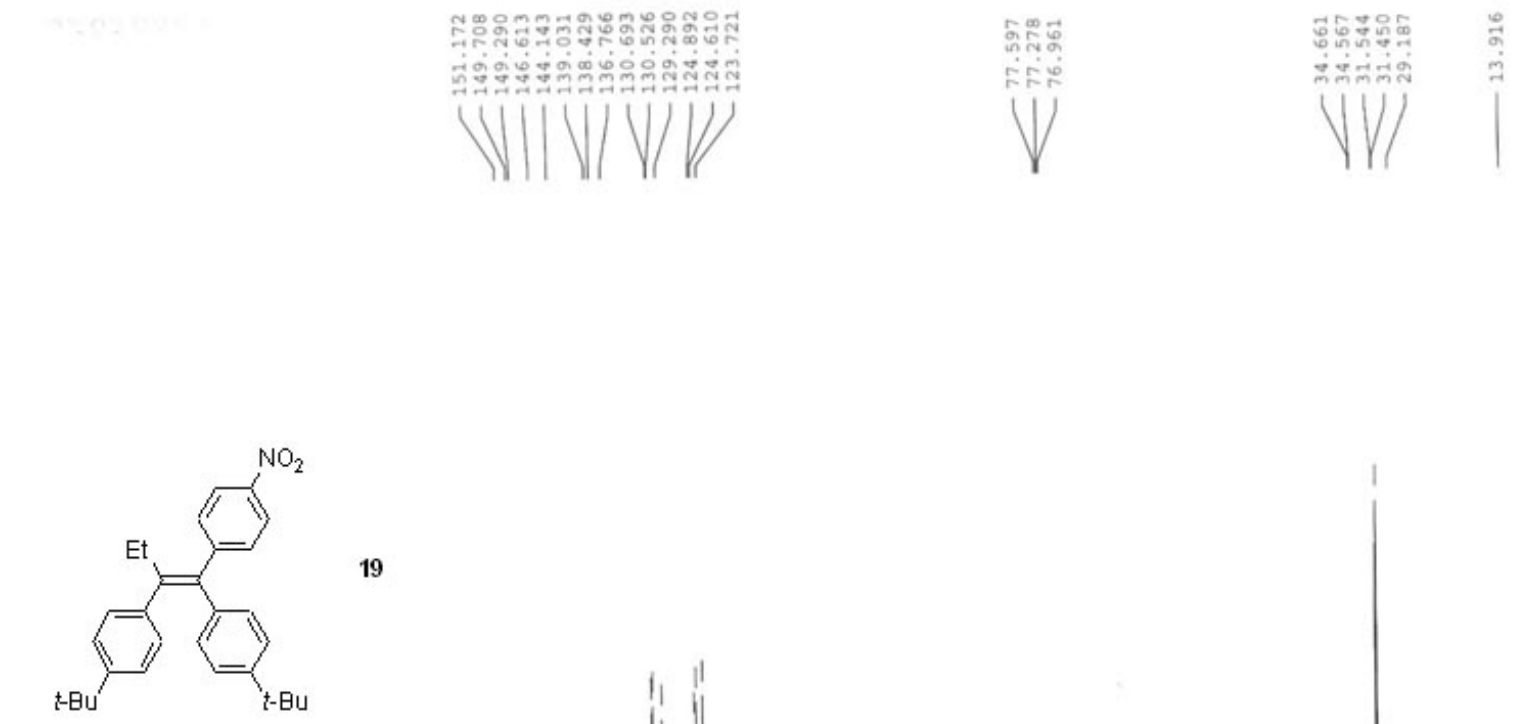

19

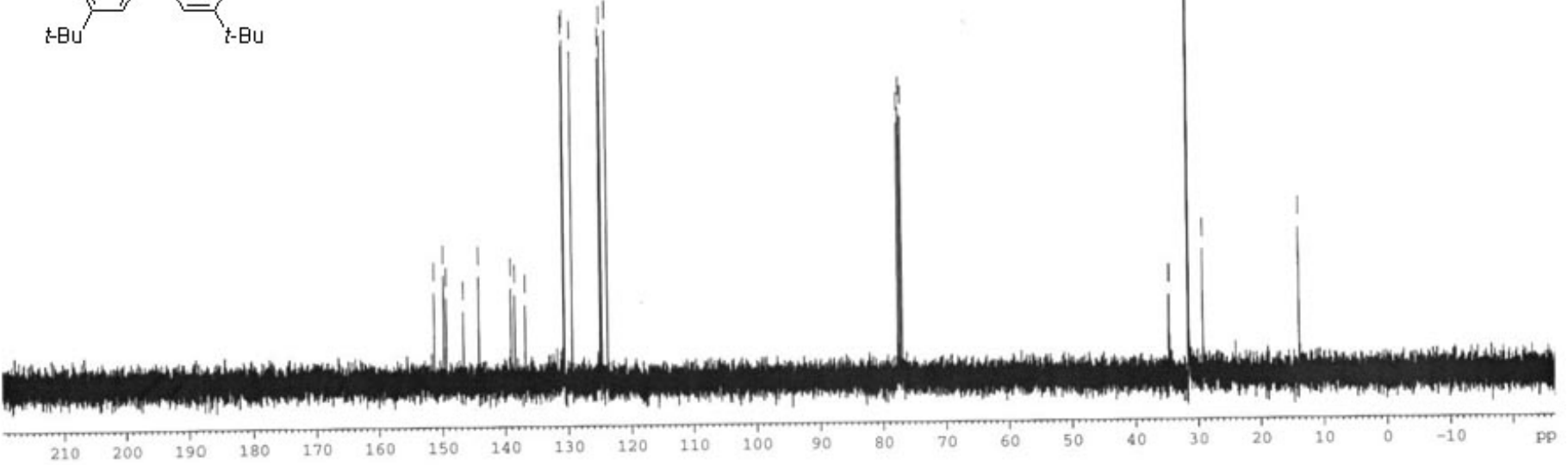



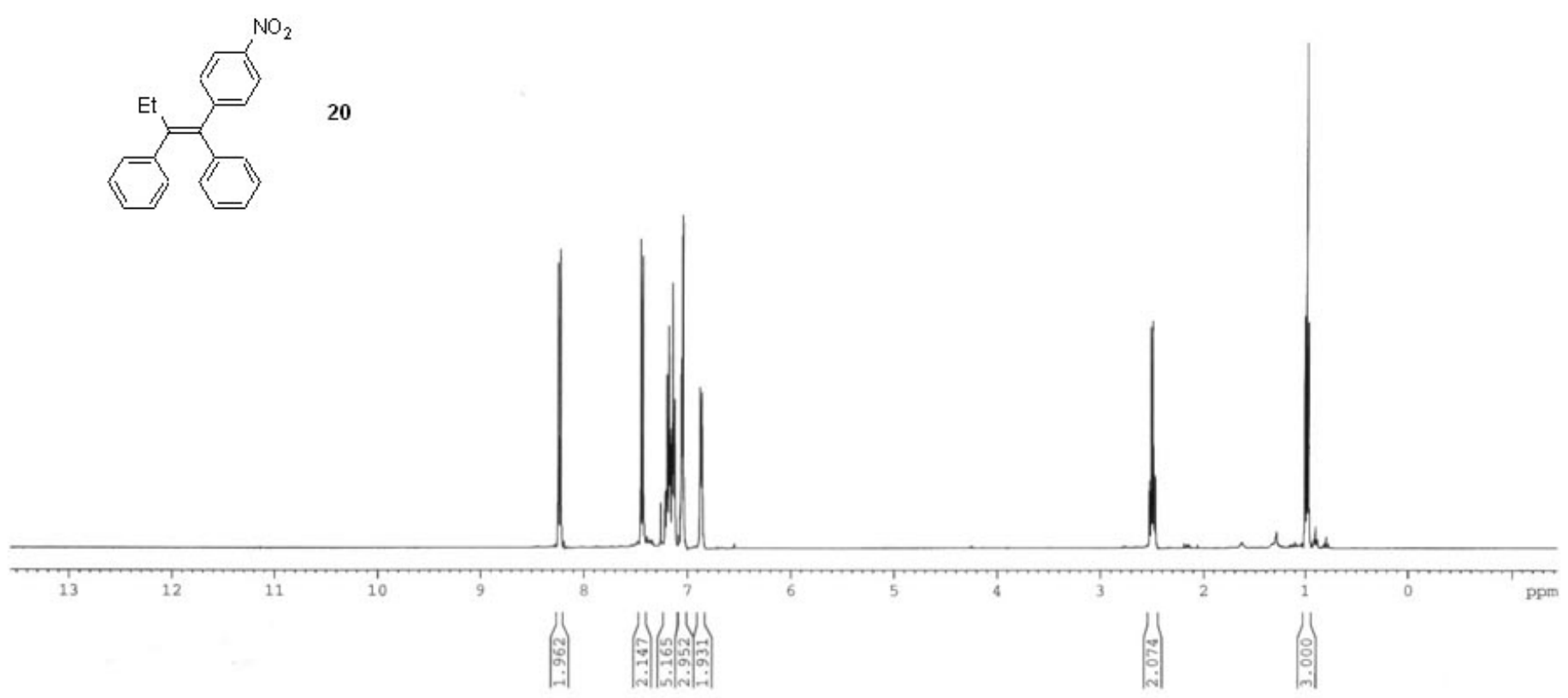


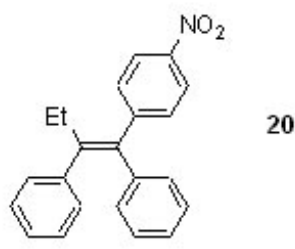

20

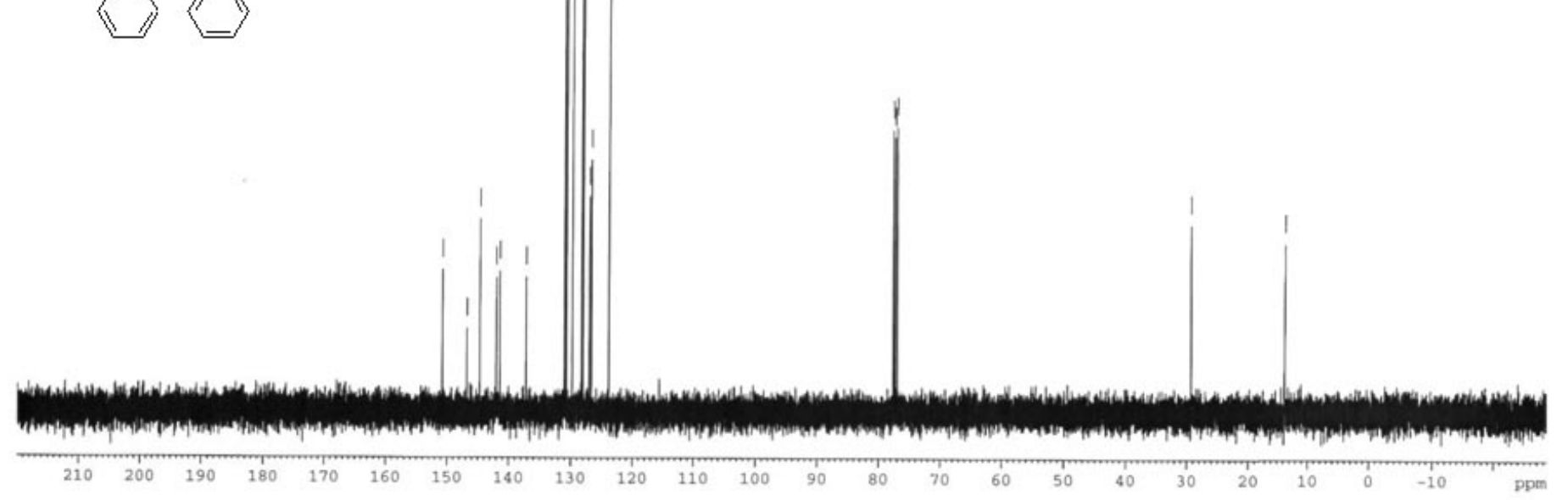




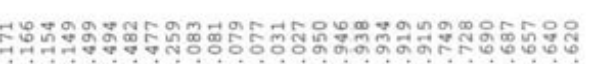
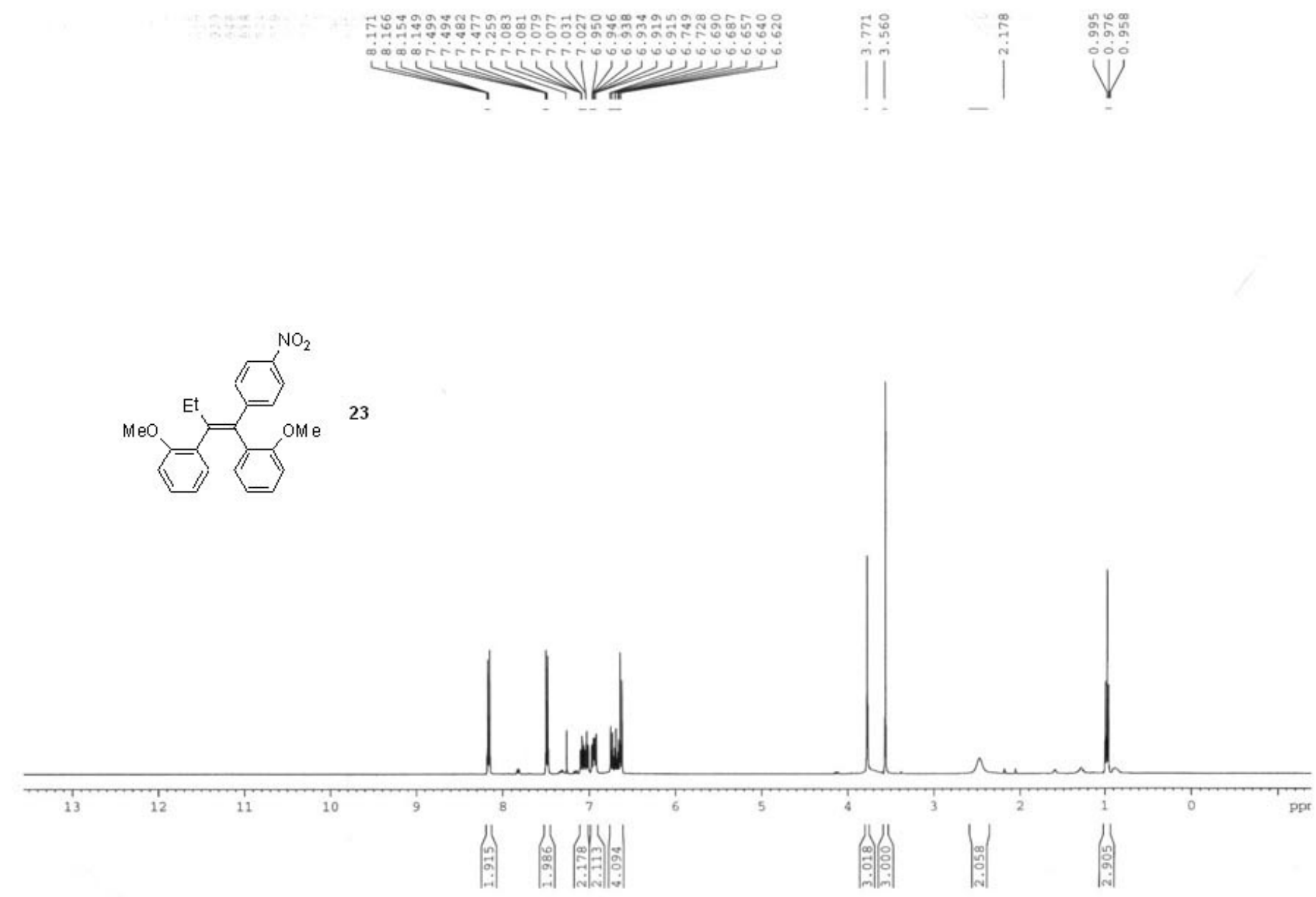

23 
도요 :

ơ

VINWWVIV
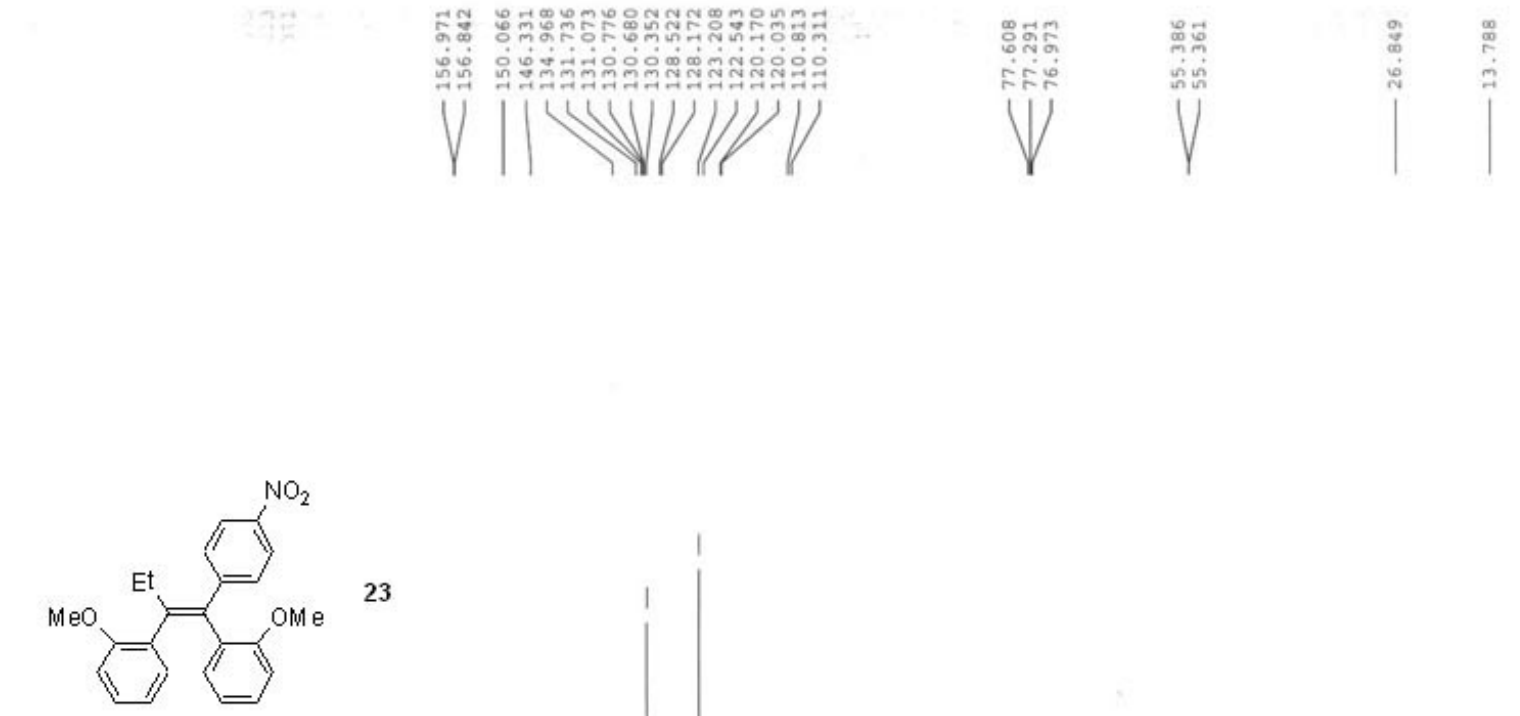


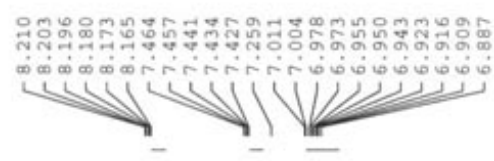

Vู|
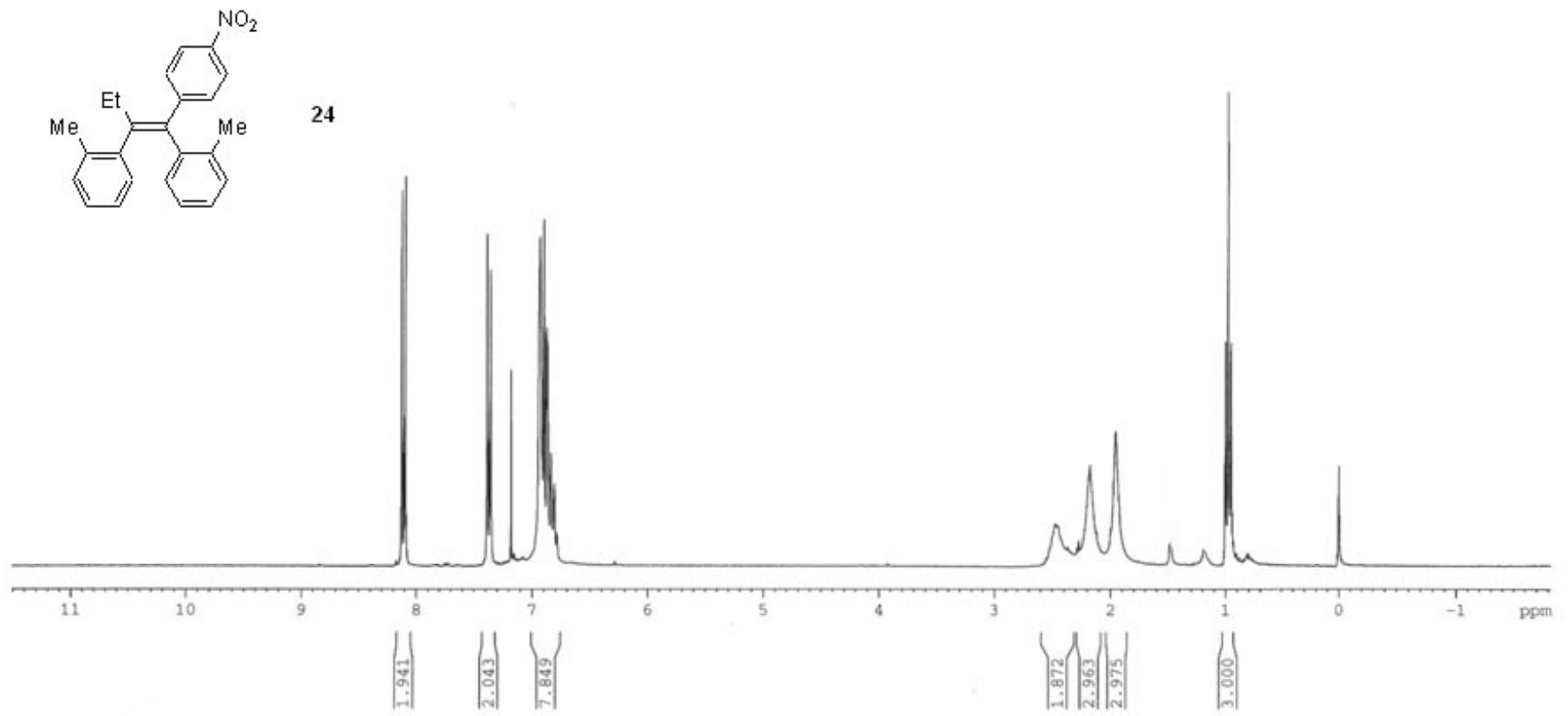

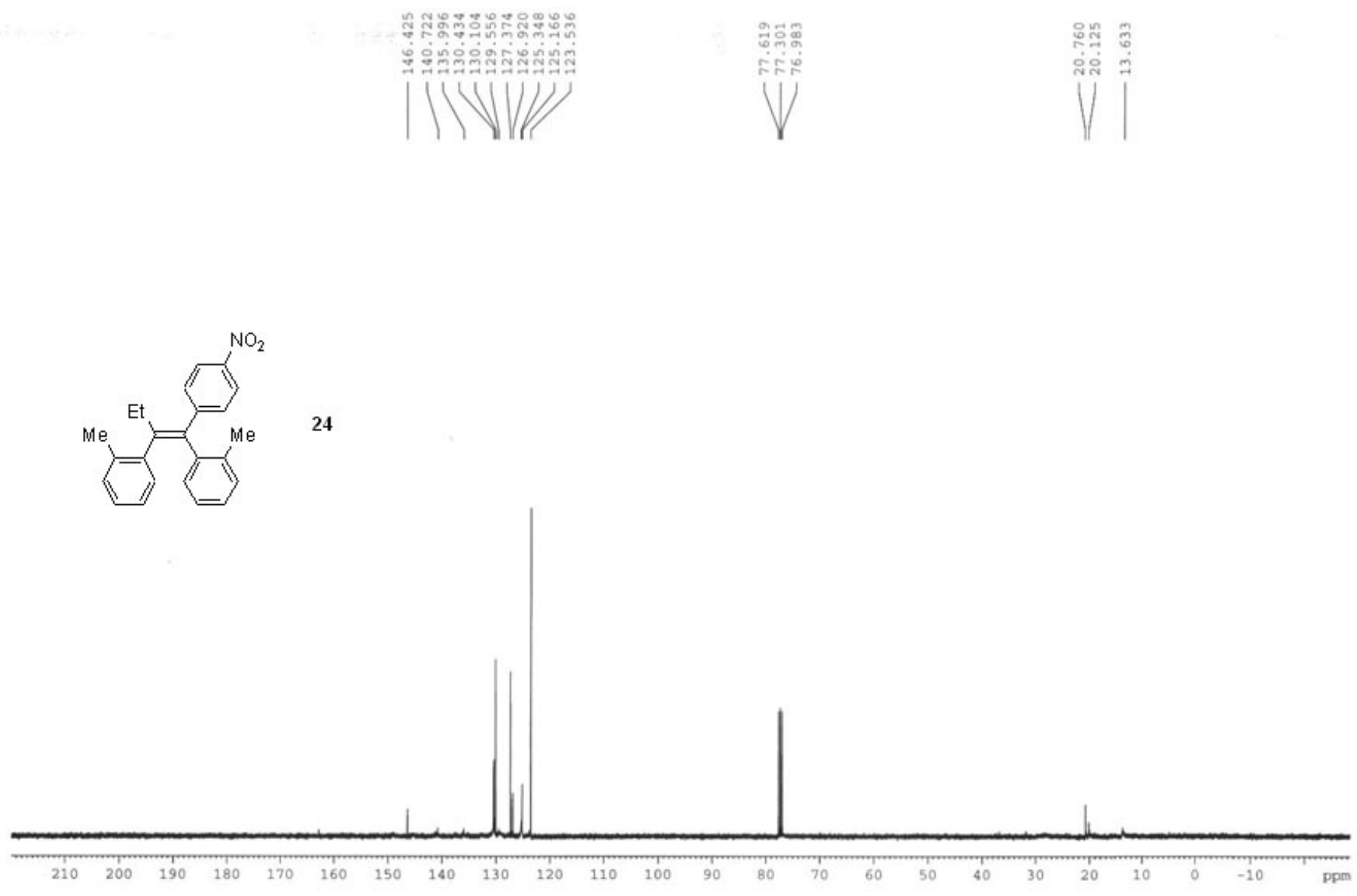


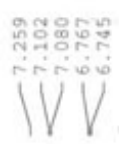

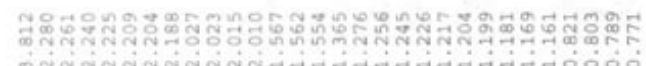

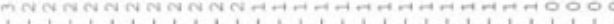

$\longrightarrow$
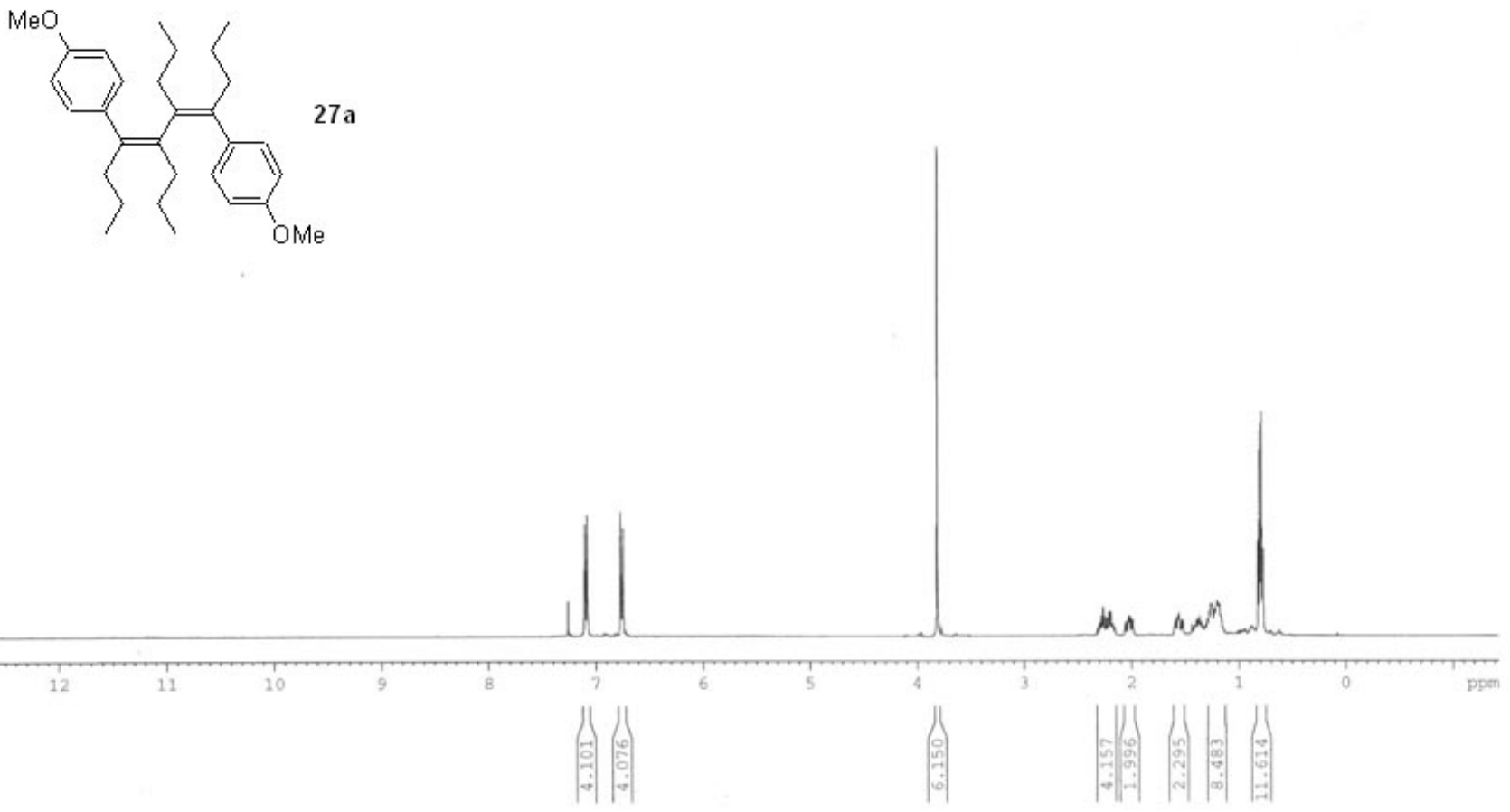


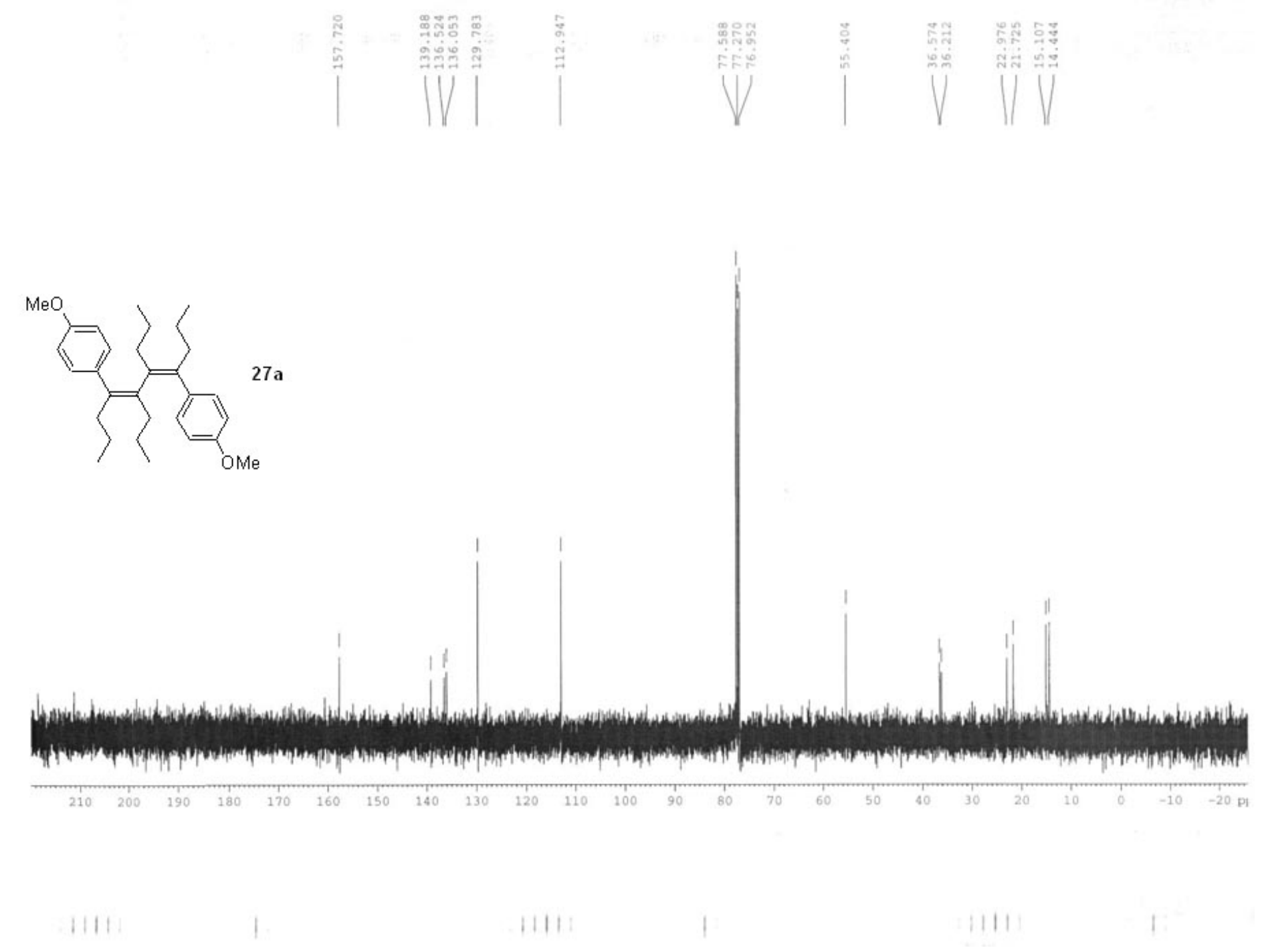




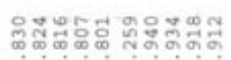

WiV IV

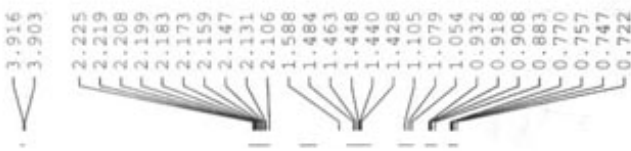

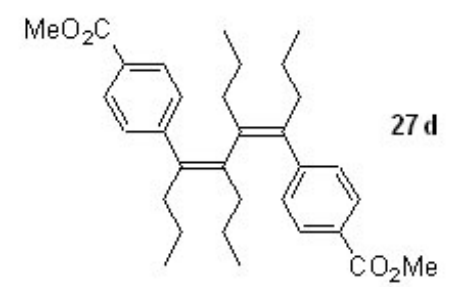

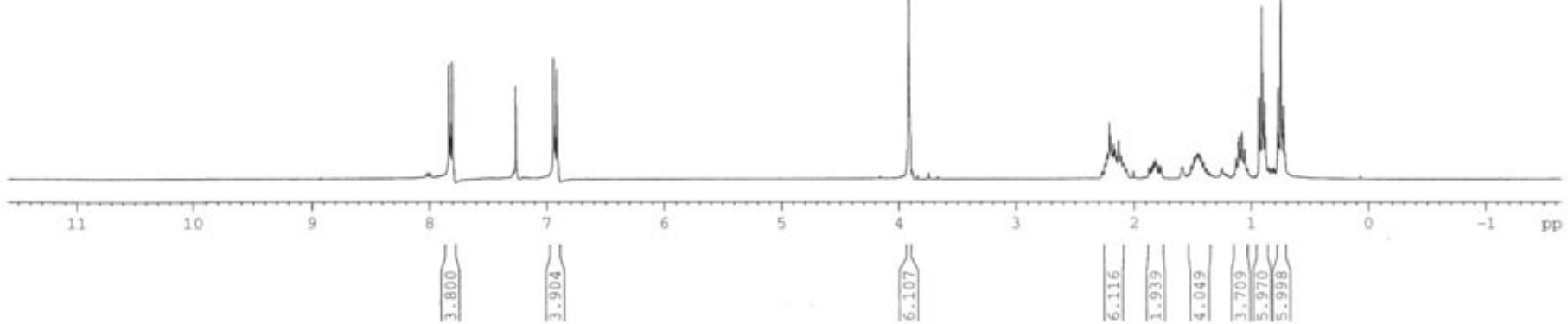

111111

1!

111111

11,

111111

111 


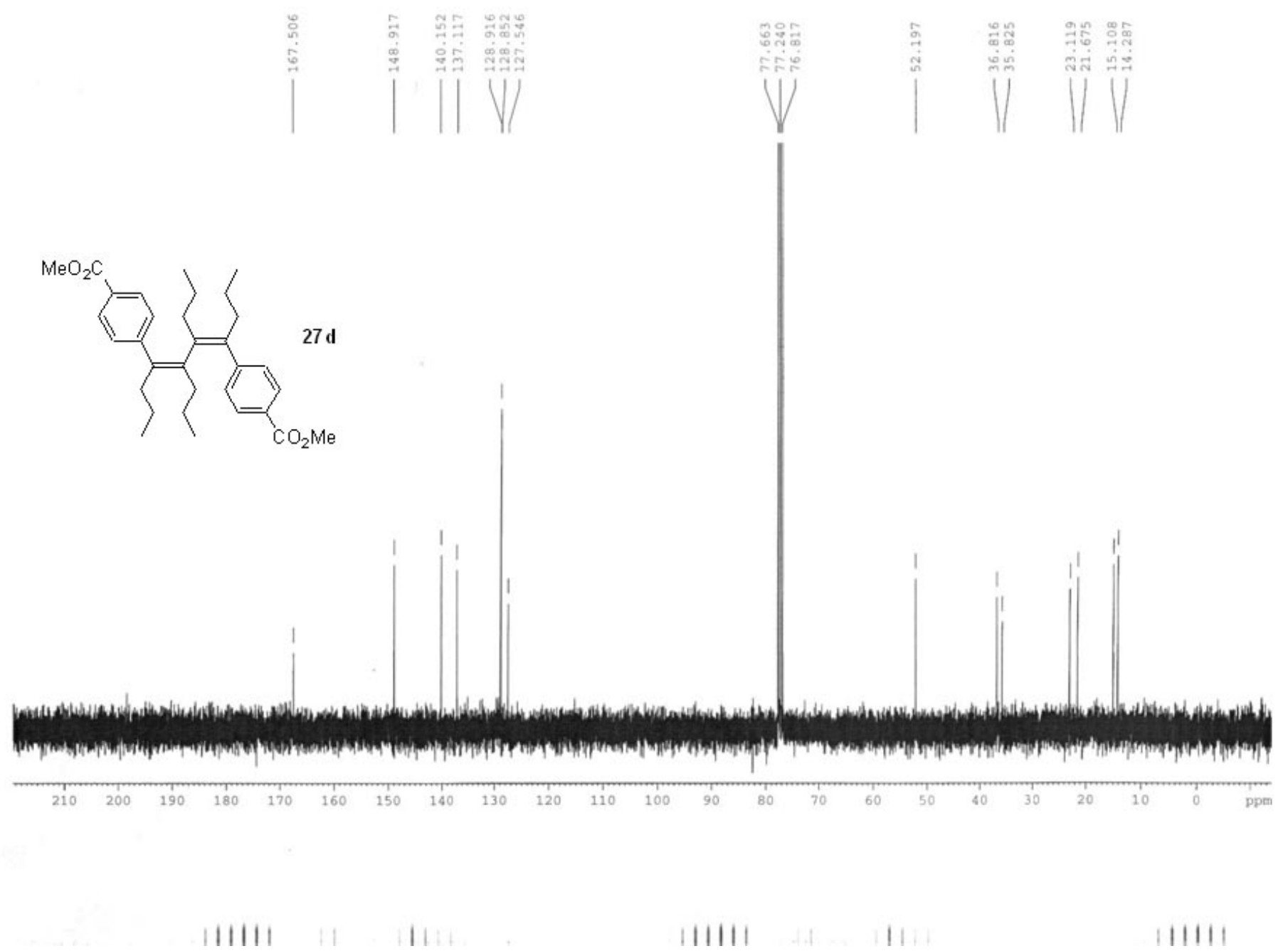




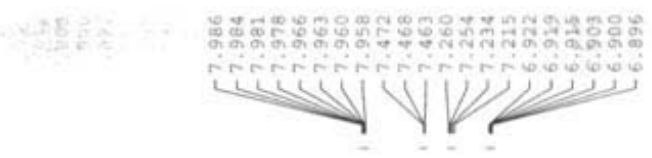

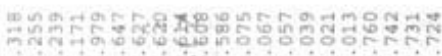

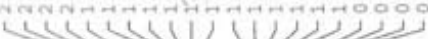
in 1
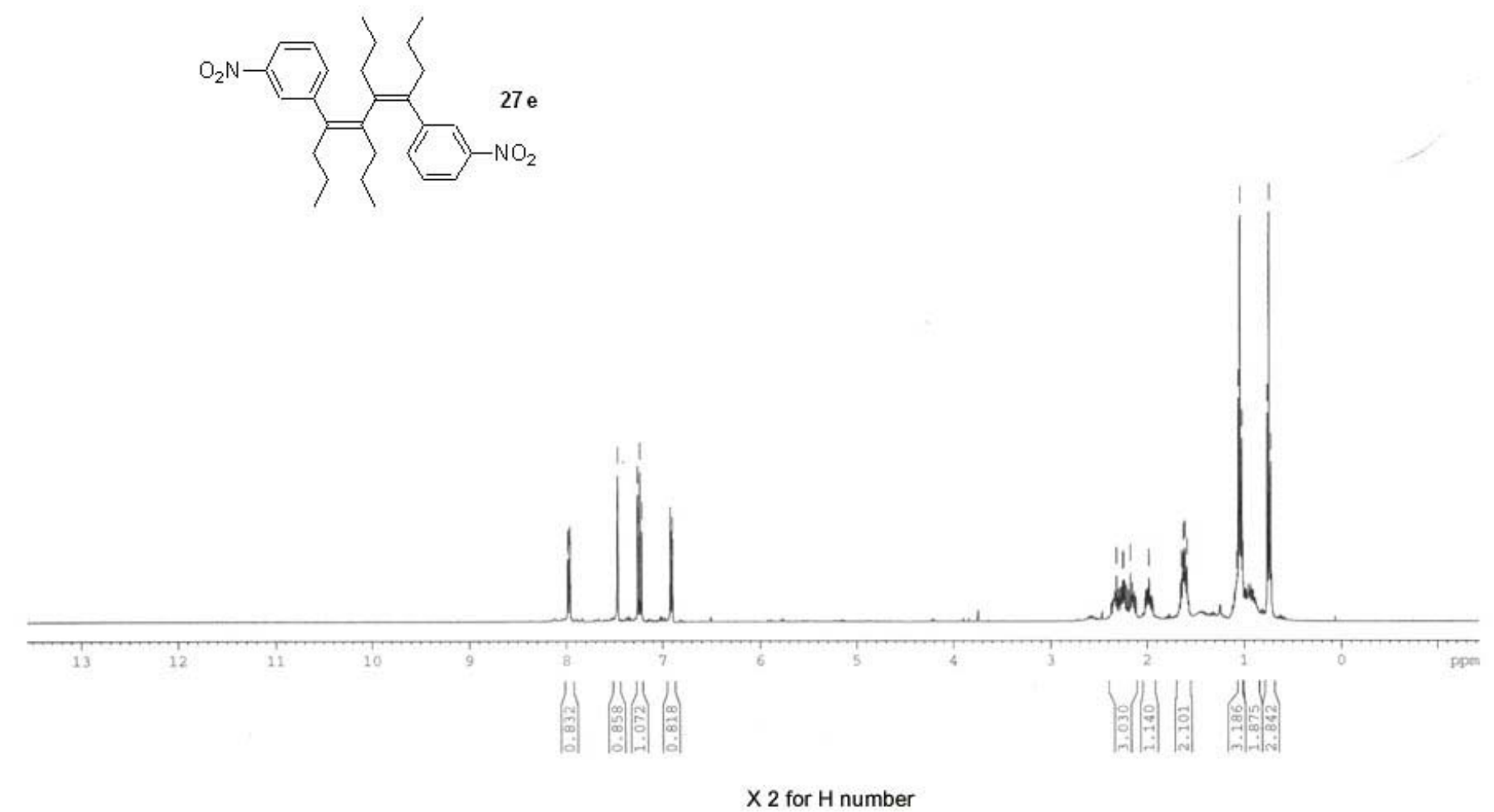

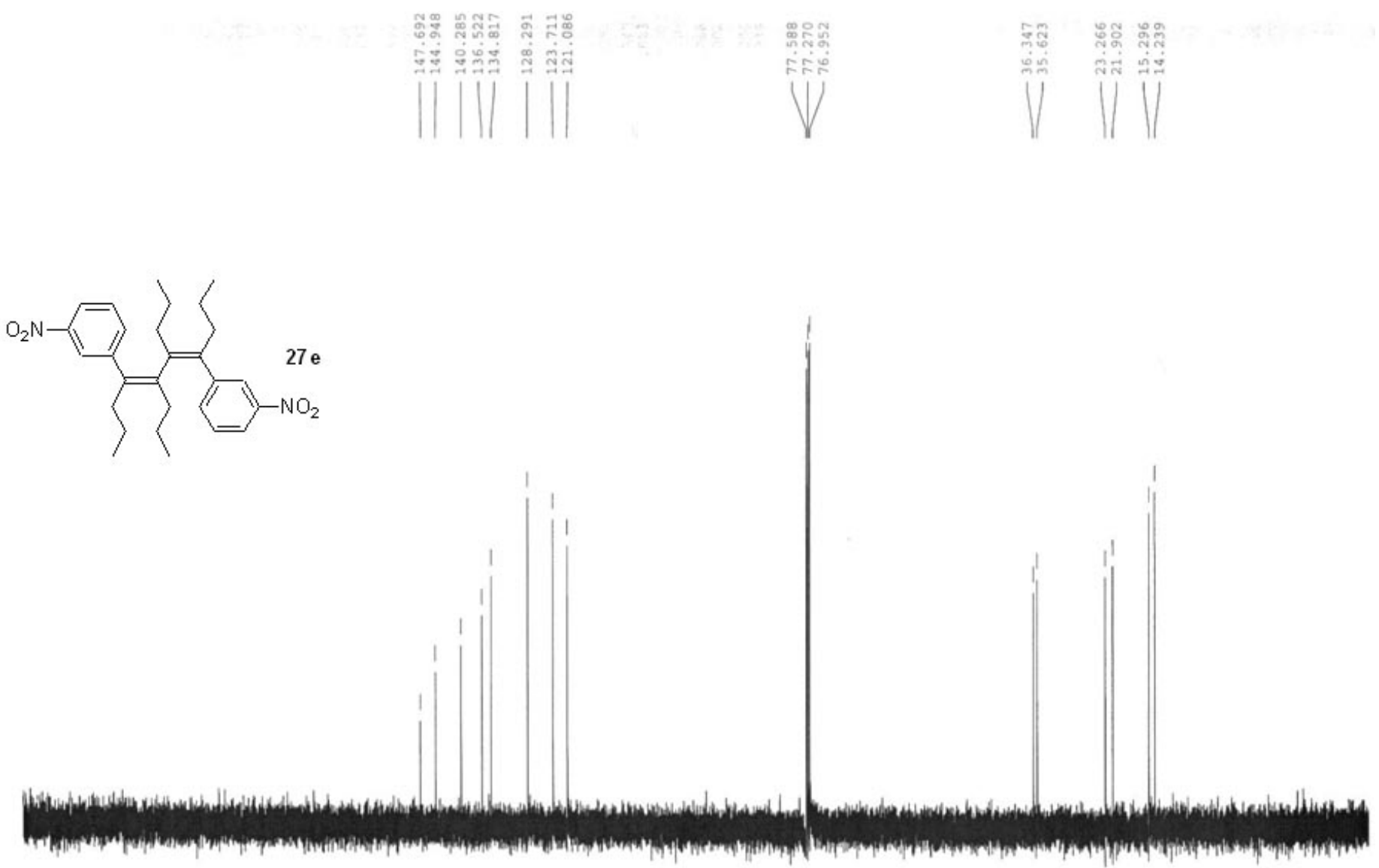
$\mathrm{MeO}$<smiles>CCC(c1ccccc1)C(c1ccccc1)c1cccc([N+](=O)[O-])c1</smiles>

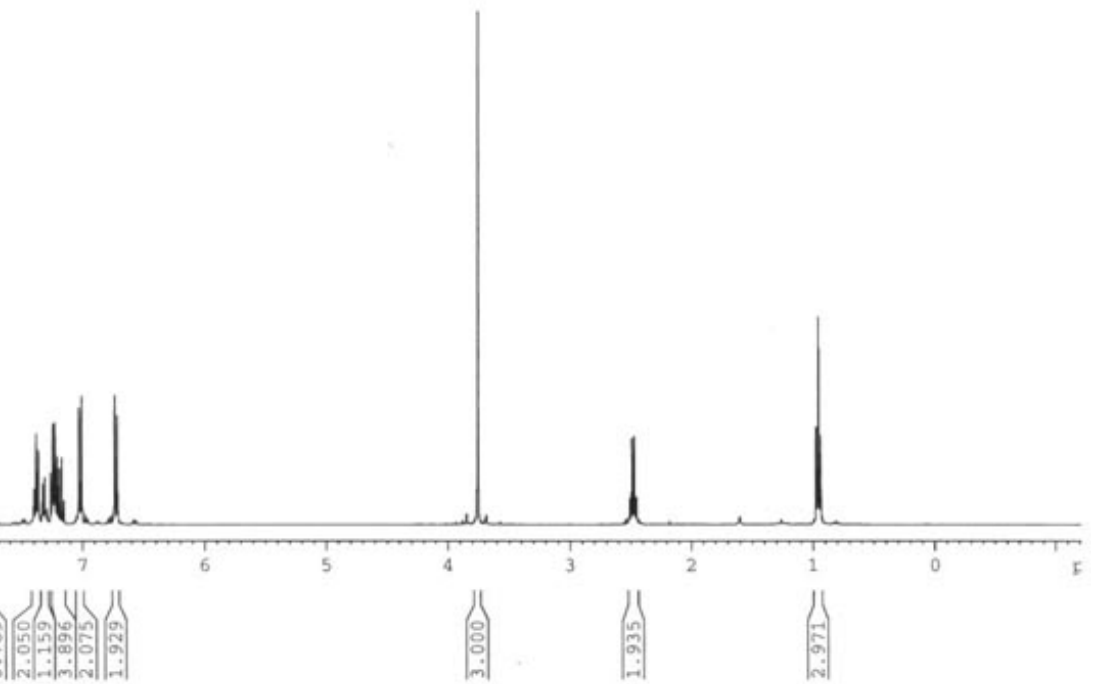



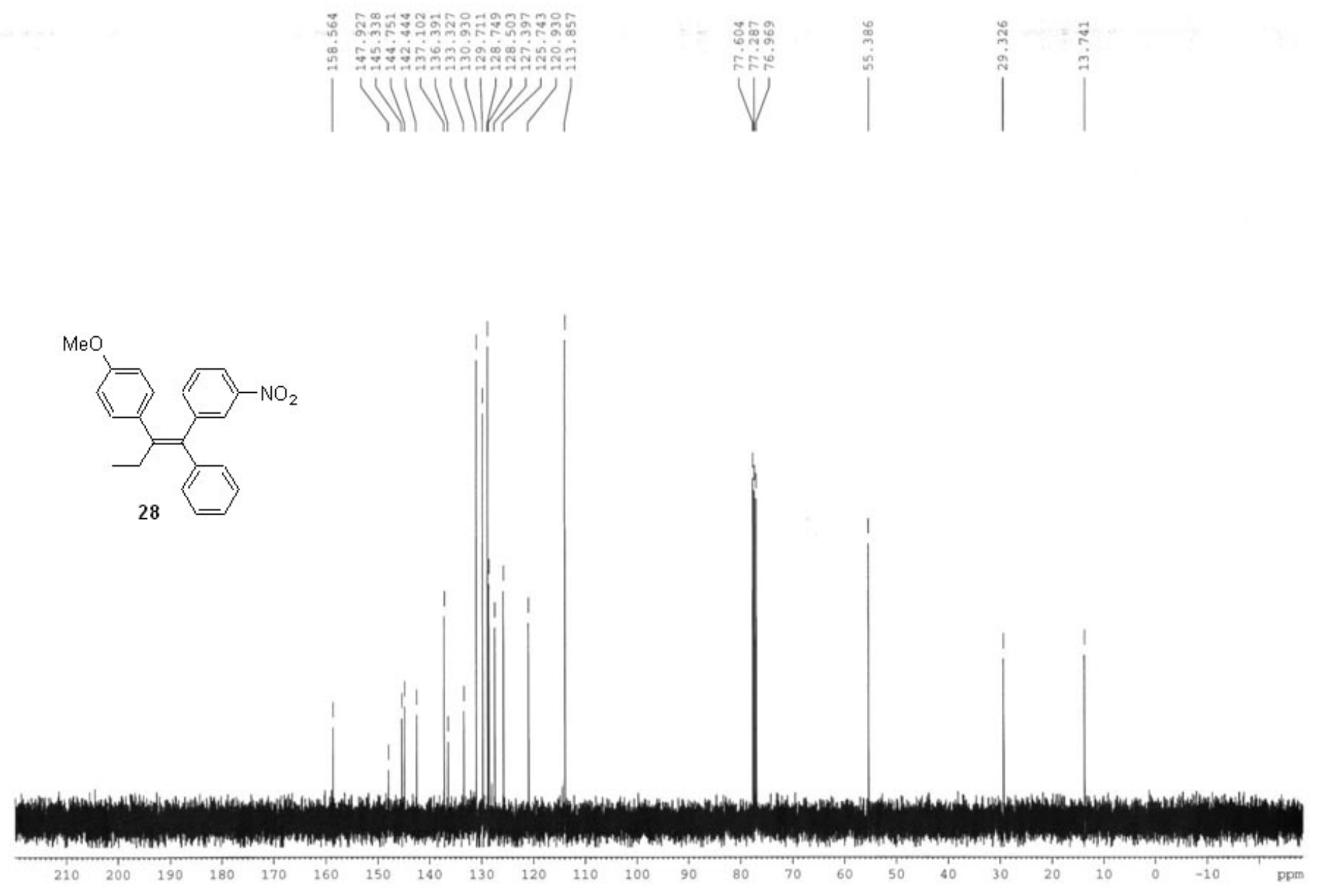